\title{
Rapid Assessment of Nonindigenous Marine Species on Coral Reefs in the Main Hawaiian Islands ${ }^{1}$
}

\author{
S. L. Coles, ${ }^{2}$ F. L. M. Kandel, ${ }^{3,4}$ P. A. Reath, ${ }^{4}$ K. Longenecker, ${ }^{2}$ and L. G. Eldredge ${ }^{2}$
}

\begin{abstract}
Coral reefs at Kaua'i, O'ahu, Moloka'i, Maui, and Hawai'i were surveyed using a rapid assessment method for marine nonindigenous and cryptogenic species commonly found in Hawaiian harbors and embayments with restricted circulation. In 41 sites surveyed by rapid assessment 26 nonindigenous and cryptogenic species (three algae, 19 invertebrates, and four fishes) were recorded from a total of 486 total taxa identified, and 17 of the nonindigenous and cryptogenic species occurred at only one or two sites. No more than six nonindigenous and cryptogenic species were recorded at any one site, and 21 of the 41 sites had fewer than three. By comparison, laboratory identification of samples collected from seven of the sites closest to harbors found 6-23 nonindigenous and cryptogenic species per site. Values for nonindigenous and cryptogenic species from rapid assessment were compared with factors potentially influencing spread and proliferation of introduced marine species. These factors included distances from harbors, boat-launching ramps, stream mouths, and shorelines; degree of shoreline urbanization; quantity of artificial surfaces in the water; reef condition and isolation from the open ocean; and native species richness. A best subsets regression model explained over $65 \%$ of the variance in nonindigenous and cryptogenic species from two predictor variables and their interaction: isolation from the open ocean and number of native taxa, with most of the variance explained by a highly significant relationship of nonindigenous and cryptogenic species with isolation from open-ocean conditions.
\end{abstract}

INTRODUCTIONS OF nonindigenous (introduced) marine species are considered to have escalated in the last $30 \mathrm{yr}$ (e.g., Carlton 1985, Carlton et al. 1990, Carlton and Geller 1993, Cohen et al. 1995, Gosliner 1995, Mills and

${ }^{1}$ This project was conducted with the financial support of the Hawai'i Coral Reef Initiative and National Oceanic and Atmospheric Administration grant no. NA03NOS4260044. This is contribution number HBS 2006-001 from the Hawai'i Biological Survey and contribution 1224 from the Hawai'i Institute of Marine Biology. Manuscript accepted 30 December 2005.

${ }^{2}$ Department of Natural Sciences, Bishop Museum, 1525 Bernice Street, Honolulu, Hawai'i 96817 (e-mail: slcoles@bishopmuseum.org).

${ }^{3}$ Department of Zoology, University of Hawai'i at Mānoa, Honolulu, Hawai'i 96822.

${ }^{4}$ Hawai'i Institute of Marine Biology, P.O. Box 1346, Kāne'ohe, Hawai'i 96744.

Pacific Science (2006), vol. 60, no. 4:483-507

(C) 2006 by University of Hawai'i Press

All rights reserved
Sommer 1995, Daehler and Strong 1996, Greenberg et al. 1996, Ruiz et al. 1997, 2000, Bax et al. 2001), sometimes with serious negative consequences when introduced species become invasive (i.e., compete with native species to the point that they alter the structure or function of the invaded ecosystem). Marine species invasions have been ranked among the most serious potential perturbations of marine ecosystems (Carlton 1994), and alteration of habitats and food webs by invasive species has been proposed as a potential major factor contributing to degradation of coral reefs (Birkeland 2004).

In Hawai' $i$ there is a substantial pool of potentially invasive marine organisms that have reached the Islands over at least the last century. Eldredge and Carlton (2002) designated 343 Hawaiian marine or brackish-water species as introduced or cryptogenic (i.e., of uncertain geographic origin sensu Chapman and Carlton [1991] and Carlton [1996]). A number of comprehensive surveys for marine 
introduced species have been completed in the Hawaiian Islands (Coles et al. 1997, 1998, 1999a,b, 2002a,b, 2004a,b, DeFelice et al. 1998, 2002), Johnston Atoll (Coles et al. 2001), American Samoa (Coles et al. 2003), Guam (Paulay et al. 2002), and port areas of northern Australia (e.g., Hewitt et al. 1998, Hoedt et al. 2000, 2001a,b, Russell and Hewitt 2000, Neil et al. 2001). Most of these studies have focused on harbors or disturbed areas with limited oceanic circulation.

Invasive introduced algae have monopolized nearshore reefs (Rodgers and Cox 1999, Woo et al. 1999, Smith et al. 2002) throughout the main Hawaiian Islands. An invasive octocoral was recently found overgrowing Hawaiian deep-water black corals (Grigg 2003, 2004), and an abundant introduced sponge may be similarly impacting shallowwater reef corals in southern Kāne'ohe Bay (Coles and Bolick 2006). An introduced, aggressive mantis shrimp has displaced native mantis shrimp species from coral rubble habitats in Hawai'i (Kinzie 1968, 1984), and reef fishes purposely introduced to Hawai' $i$ in the 1950s are common to abundant on reefs throughout the Hawaiian archipelago (Randall and Kanayama 1972, Randall 1987). However, despite the potential importance of invasive introduced species on coral reefs, little is known about the occurrence or impact of most marine introductions in Hawai' $\mathrm{i}$ or elsewhere in the tropical Pacific (Coles and Eldredge 2002). Further, there has been no evaluation of the factors that may influence the establishment and proliferation of introduced species on coral reefs.

We used a standardized rapid assessment technique to determine the presence of introduced marine species on coral reefs for the five largest Hawaiian Islands and evaluated factors that may influence introduced species occurrence. Previous findings (Coles et al. 1997, 1998, 1999a,b, 2001, 2002a,b, 2003, DeFelice et al. 1998, 2002) suggested that three principal factors may influence the distribution and proliferation of most nonindigenous species in Hawai' $i$ and other tropical areas: (1) isolation may limit recruitment from likely sources such as harbors and boat landings where introductions are mostly likely to occur, (2) oligotrophic open-ocean reef environments may not provide sufficient food to support the filter-feeding invertebrates that prevail among nonindigenous organisms, (3) generally higher native species richness on ocean-exposed coral reefs may act to limit introduced species that proliferate in the less-diverse communities of harbors or estuaries. Few introduced species have been noted in higher-diversity tropical regions for ports in northern Australia (Hewitt et al. 1998, Hoedt et al. 2000, 2001a,b, Russell and Hewitt 2000, Neil et al. 2001), Guam (Paulay et al. 2002), and at American Samoa (Coles et al. 2003), where native species richness is substantially higher than in Hawai'i.

In this study we compared the occurrence of introduced species among 41 coral reef sites in various environmental conditions and distances from harbors, piers, and boat ramps throughout the main Hawaiian Islands. We evaluated the results in terms of important factors that may influence the occurrence of introduced species at the reef sites.

\section{MATERIALS AND METHODS}

\section{Field Techniques}

A total of 41 coral reef sites distributed across five islands was surveyed using a rapid assessment technique. Sites were selected to include a variety of environmental characteristics that might influence the establishment of introduced species, such as proximity to harbors and boat ramps, distance from shore or streams, presence of artificial structures in the water or degree of shoreline development, reef condition, and exposure to the open ocean. Selection criteria also included utilization of sites established by ongoing reef-monitoring programs that could provide historical and future data on reef communities. Twenty-four of the 41 rapid assessment sites coincided with Hawai'i Coral Reef Assessment and Monitoring Program (CRAMP) sites (Jokiel 2002, Brown et al. 2004), and eight of the 10 surveys on Hawai'i were made at West Hawai'i Aquarium Project (WHAP) sites (Tissot et al. 2001).

Anticipating that introduced species were 


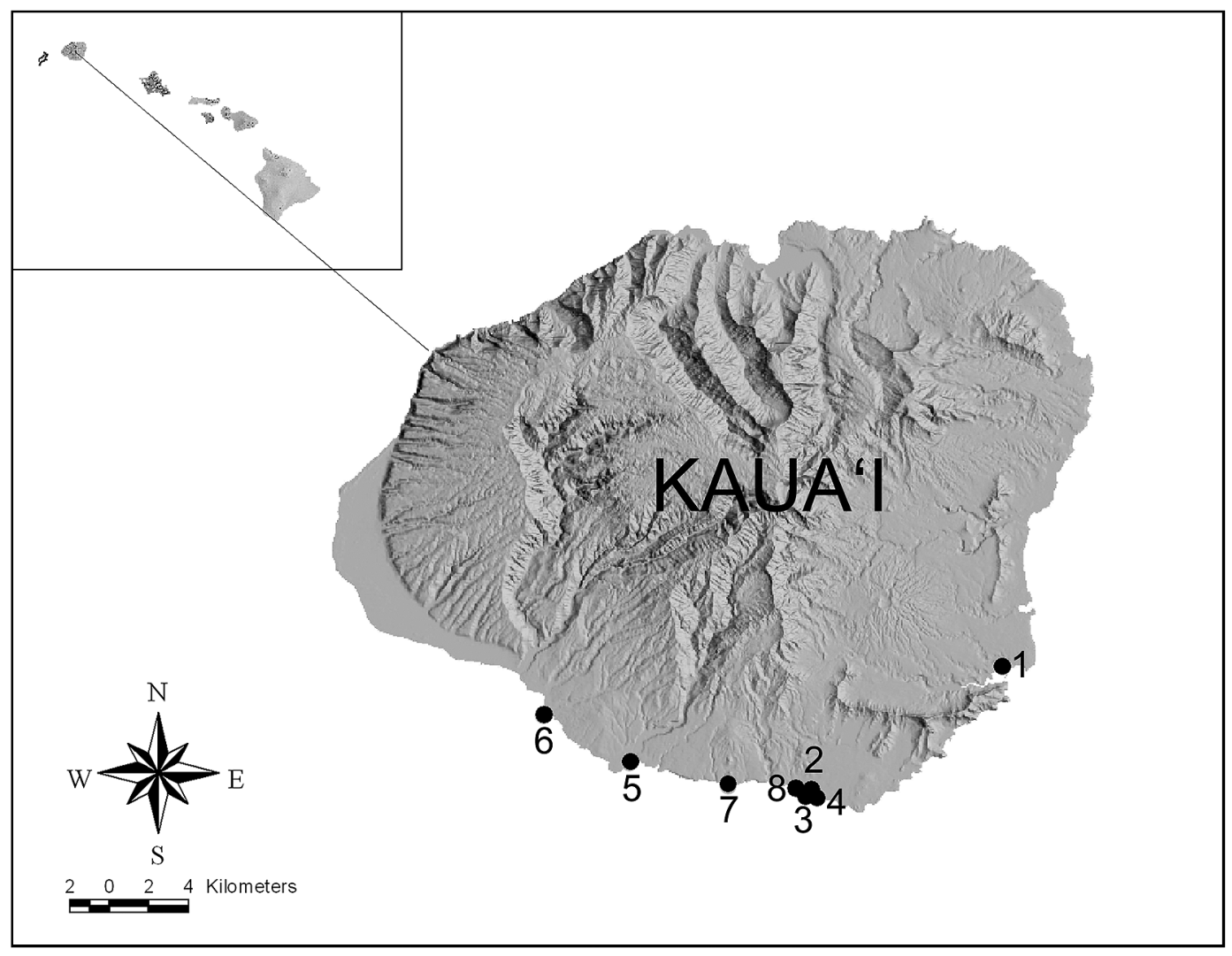

Figure 1. Kaua'i coral reef stations.

likely to be low in frequency and abundance, we utilized a replicable methodology to examine large reef areas within nodecompression time limits for up to three scuba dives per day at 10-20 m. Locations of the 41 sites surveyed are shown in Figures $1-5$, and their station numbers, locations, depth ranges, and coordinates are listed in Table 1. Our timed search approach provided a standardized and sufficiently large search area to assure that most macrobiota at the site were observed. We recorded the location of each reef site using Global Positioning System and deployed a 50-m transect line parallel to the shore for $25 \mathrm{~m}$, then turned the line at a right angle for the remaining 25 $\mathrm{m}$, with the resulting triangular observation area established by the hypotenuse thus approximating $312 \mathrm{~m}^{2}$. Two observers (S.L.C. and F.L.M.K.) swam in tandem for $15 \mathrm{~min}$ along the perimeter of the triangle, recording the first occurrence of all invertebrates, fishes, and identifiable macroalgae along a swath up to $2 \mathrm{~m}$ on either side. For the next $15 \mathrm{~min}$ we recorded organisms that occurred within the $312-\mathrm{m}^{2}$ triangular area. Finally, we spent 15 min searching outside the triangle recording all taxa not previously observed. In addition to the observations made by these two observers, a third diver (P.A.R.) searched subhabitats known to support nonindigenous and cryptogenic species (i.e., overhangs, crevices, dead coral heads, coral rubble, algal turf and tufts) at 28 sites on Kaua'i, Moloka'i, Maui, and Hawai'i (Table 1) and recorded cryptic organisms. We recorded all field-identifiable organisms on underwater paper, and we collected species suspected to be introduced and 


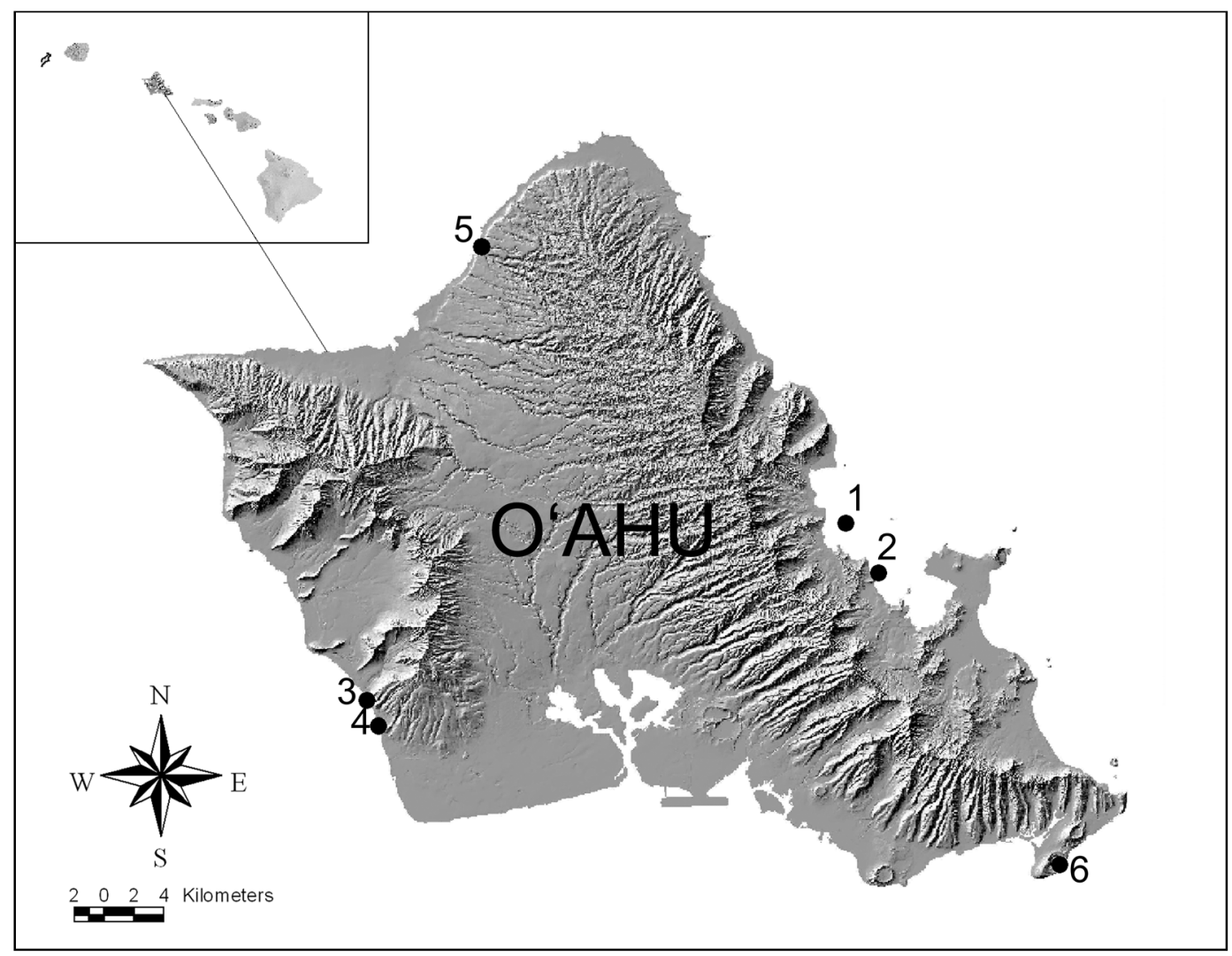

Figure 2. O'ahu coral reef stations.

transported them to the laboratory for identification and/or verification by taxonomic experts. We designated species as nonindigenous, cryptogenic, or native according to J. T. Carlton and L.G.E. (unpubl. data) and the Checklist of the Marine Invertebrates of the Hawaiian Islands (http://www2 .bishopmuseum.org/HBS/invert/list_home .htm). Taxa not identified to species were designated native unless they were previously known unnamed introductions (e.g., Ascidia sp. A [Abbott et al. 1997; J. T. Carlton and L.G.E., unpubl. data]). New reports for $\mathrm{Ha}-$ wai' $i$ were designated cryptogenic or "of uncertain origin that is neither demonstrably native or introduced" (Carlton 1996:1653). We consider this an appropriate category for new species reports until sufficient information is available to verify their introduced or native status and the degree to which they are widespread in Hawai'i. This approach resulted in conservative estimates for the numbers of known or possible introductions for each site.

\section{Evaluation of Rapid Assessment Technique}

Our visual rapid assessment technique did not include minute organisms such as amphipods, isopods, tanaids, and small hydroids that are a major component of the introduced species community but can only be identified by microscopic examination. However, the observations included introduced macrobiota such as algae, fishes, and invertebrates such as sponges, larger hydroids, decapods, bryozoans, and tunicates. To evaluate the sensitivity of the rapid assessments for determining 


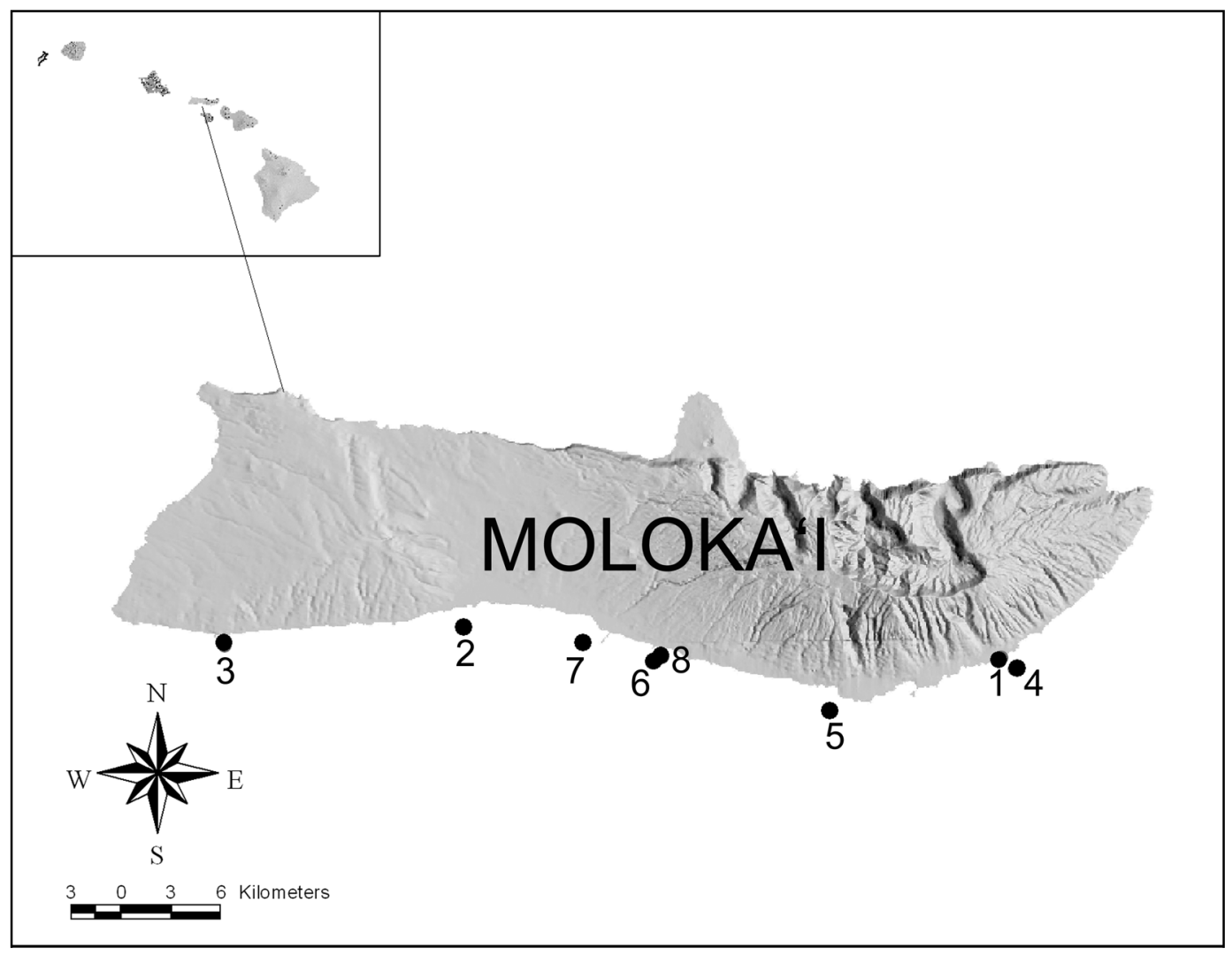

Figure 3. Moloka'i coral reef stations.

all nonindigenous and cryptogenic species, one of the field team (P.A.R.) made systematic collections of benthic organisms at seven stations on islands other than O'ahu (Table 1). These sites were on reefs closest to the main interisland shipping ports of Kaua' $i$ (KA1), Moloka'i (MO7), and Hawai'i (HA1 and HA10) or adjacent to small-craft harbors (KA5, MO3, and MA7). Samples were collected opportunistically as a composite sample from all available subhabitats within the reef in the vicinity but outside the $312-\mathrm{m}^{2}$ triangle (i.e., bases of coral heads, coral rubble macroalgae, and turf algae, and from cryptic areas under ledges and in crevices) and preserved in $70 \%$ ethanol. These organisms $>0.5 \mathrm{~mm}$ were identified in the laboratory to species or the lowest practical taxon. These results were compared with those obtained from rapid assessment surveys at the same sites.

\section{Variables Influencing Introductions}

A number of both natural and anthropogenic factors may influence the introduction, distribution, and proliferation of nonindigenous marine species. We evaluated nine potential contributing factors as part of this study: (1) Hawaiian harbors have a large complement of introduced species (Coles et al. 1997, $1999 a, b, 2004 b)$ and proximity to harbors may increase the probability of occurrence of introduced species on reefs. (2) Similarly, small boats may inadvertently transfer introduced species, thus proximity to boatlaunching areas may also be a factor in spreading introductions. (3) Flooding and 


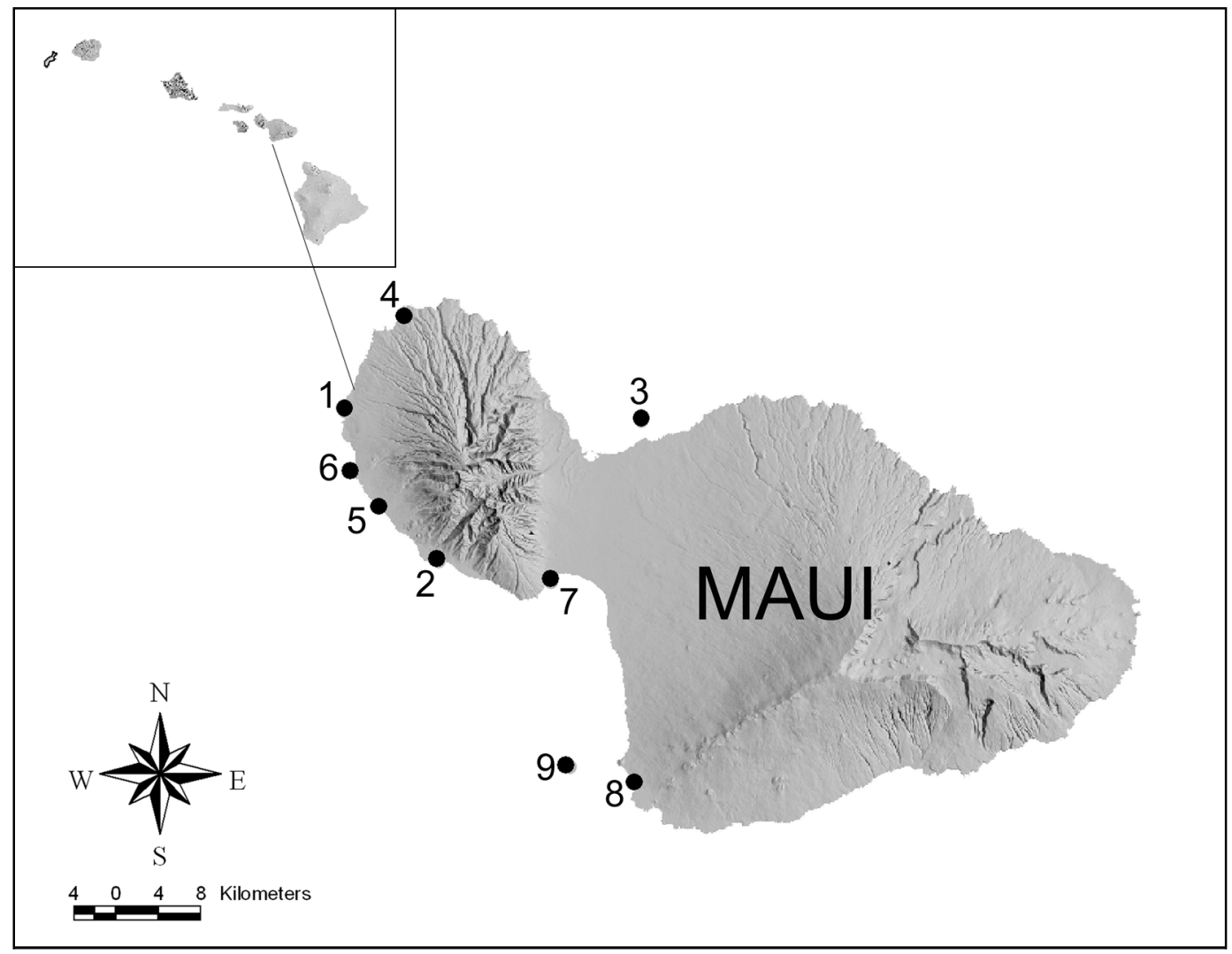

Figure 4. Maui coral reef stations.

sedimentation from streams may result in a stressed environment more conducive to establishment of introduced species on denuded or disturbed reef surfaces. Studies in estuaries of $\mathrm{O}^{\prime}$ ahu (Englund et al. 2000) showed a high incidence of introduced species. Therefore, proximity to stream mouths was evaluated as a factor potentially influencing nonindigenous and cryptogenic species. (4) Some introduced species, especially alien algae and the invertebrates that occur with them, are most abundant along or near shorelines, and distance from shoreline was evaluated as a possible influence on introductions. We used Geographic Information System-based maps (Figures 1-5) to determine the distances of these four features from each survey site.

Other potential determining factors con- sidered were as follows: (5) the amount of artificial substrate in the water or along the shoreline; (6) the degree to which the adjacent shoreline was urbanized; (7) the degree to which the surveyed area was isolated from the open ocean; and (8) the general condition of the coral reef itself, again on the assumption that a disturbed reef was more susceptible to introductions and invasions. We ranked, on site, the condition of these latter four factors by the criteria in Table 2. Finally, based on previous observations that numbers of introduced species appeared to be inversely correlated with the numbers of native species (Coles et al. 1997, Coles et al. 1998, $1999 a, b, 2002 a, b$ ), we included (9) the number of native taxa, or native species richness, at each site as a predictive variable in our analyses. 


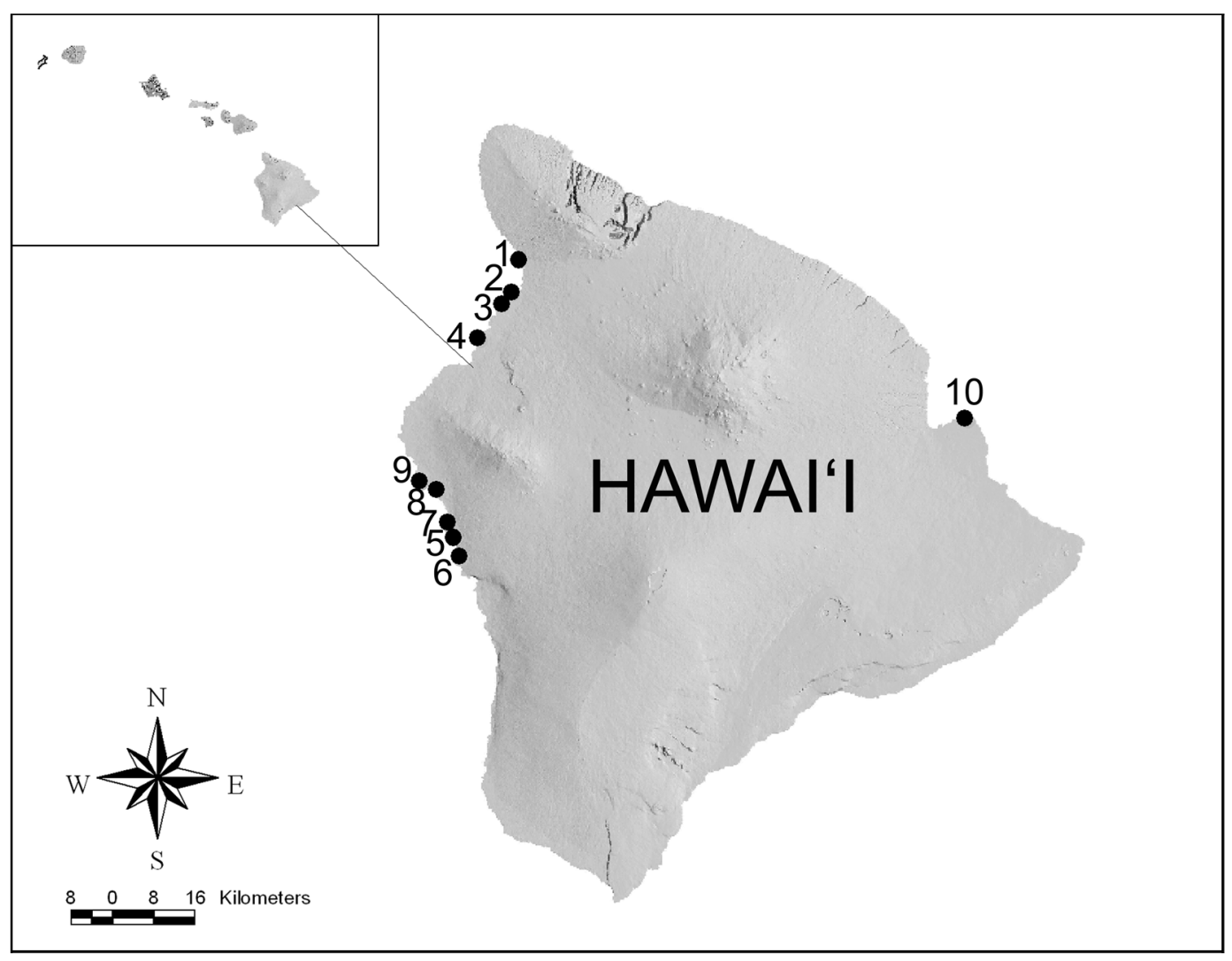

Figure 5. Hawai'i coral reef stations.

\section{RESULTS}

Numbers of Nonindigenous and Cryptogenic Species and Total Taxa

Using rapid assessment, we identified a total of 486 taxa, ranging from a maximum of 151 taxa at Station 6 on Hawai'i to a minimum of 40 taxa at Station 2 on Kaua'i and at Station 3 on Moloka'i. All taxa identified by rapid assessment at the 41 sites are listed in Appendix B of the full report (Coles et al. 2004a). The numbers of total taxa recorded by rapid assessment at each station are shown in Figure 6. Of these, 26 were cryptogenic or introduced species (collectively termed nonindigenous and cryptogenic species), comprising approximately $5 \%$ of all taxa identified in rapid assessment surveys. Distri- bution of the 26 nonindigenous and cryptogenic species among stations surveyed is shown in Table 3. Most nonindigenous and cryptogenic species occurred infrequently among the 41 stations, with only eight species recorded at more than four sites. The most frequent nonindigenous and cryptogenic species were the fishes roi (Cephalopholis argus Wooster, 23 sites) and ta'ape (Lutjanus kasmira (Forsskål), 10 sites), the conical hoof shell Hipponix australis Lamarck (17 sites), the didemnid ascidian Didemnum cf. candidum Savigny (14 sites), the spiny alga Acanthophora spicifera (Vahl) Børgesen (7 sites), the sponge Mycale cf. armata Thiele (5 sites), the Christmas tree hydroid Pennaria disticha (Goldfuss) (5 sites), and the feather duster worm Sabellastarte spectabilis (Grube) (5 sites). The remain- 
TABLE 1

Locations, Depths, and Coordinates for Stations Surveyed

\begin{tabular}{|c|c|c|c|c|c|c|}
\hline Island & Station & Code & Location & Depth (m) & Latitude $\mathrm{N}$ & Longitude $W$ \\
\hline \multirow[t]{8}{*}{ Kaua'i } & $1^{* \dagger}$ & KA1 & Marriott Hotel Reef & $2.5-6$ & $21^{\circ} 57^{\prime} 36.3^{\prime \prime}$ & $159^{\circ} 21^{\prime} 14.6^{\prime \prime}$ \\
\hline & 2 & KA2 & Beach House Reef & $1-1.5$ & $21^{\circ} 53^{\prime} 5.9^{\prime \prime}$ & $159^{\circ} 28^{\prime} 45.5^{\prime \prime}$ \\
\hline & 3 & KA3 & Ho'ai Bay & $7.5-10.5$ & $21^{\circ} 52^{\prime} 48.7^{\prime \prime}$ & $159^{\circ} 28^{\prime} 28.3^{\prime \prime}$ \\
\hline & 4 & KA4 & Ho'ai Bay & $2.5-3.0$ & $21^{\circ} 52^{\prime} 53.4^{\prime \prime}$ & $159^{\circ} 28^{\prime} 34.2^{\prime \prime}$ \\
\hline & $5^{* \dagger}$ & KA5 & Port Allen Harbor & $7.5-10.5$ & $21^{\circ} 54^{\prime} 3.5^{\prime \prime}$ & $159^{\circ} 35^{\prime} 49.9^{\prime \prime}$ \\
\hline & 6 & KA6 & “Tiger's" & $9.0-11.0$ & $21^{\circ} 55^{\prime} 45.5^{\prime \prime}$ & $159^{\circ} 39^{\prime} 11.5^{\prime \prime}$ \\
\hline & 7 & KA7 & Nōmilu Pond & $7.5-9.0$ & $21^{\circ} 53^{\prime} 13.7^{\prime \prime}$ & $159^{\circ} 31^{\prime} 58.8^{\prime \prime}$ \\
\hline & 8 & KA8 & Kukui'ula & $6.5-7.5$ & $21^{\circ} 53^{\prime} 8.4^{\prime \prime}$ & $159^{\circ} 29^{\prime} 16.8^{\prime \prime}$ \\
\hline \multirow[t]{6}{*}{ O'ahu } & $1^{\dagger}$ & OA1 & Kāne'ohe Bay, Waiāhole & $1.0-5$ & $21^{\circ} 28^{\prime} 35.2^{\prime \prime}$ & $157^{\circ} 49^{\prime} 55.0^{\prime \prime}$ \\
\hline & $2^{\dagger}$ & $\mathrm{OA} 2$ & Kāne'ohe Bay, He‘eia & $0.5-6$ & $21^{\circ} 26^{\prime} 48.0^{\prime \prime}$ & $157^{\circ} 48^{\prime} 37.0^{\prime \prime}$ \\
\hline & $3^{\dagger}$ & OA3 & Nānākuli Point & $4-5$ & $21^{\circ} 22^{\prime} 20.3^{\prime \prime}$ & $158^{\circ} 08^{\prime} 32.1^{\prime \prime}$ \\
\hline & $4^{\dagger}$ & OA4 & Kahe Beach Park & $3-4$ & $21^{\circ} 21^{\prime} 34.1^{\prime \prime}$ & $158^{\circ} 08^{\prime} 6.1^{\prime \prime}$ \\
\hline & $5^{\dagger}$ & OA5 & Pūpūkea & $1-6$ & $21^{\circ} 38^{\prime} 46.6^{\prime \prime}$ & $158^{\circ} 03^{\prime} 52.4^{\prime \prime}$ \\
\hline & $6^{+}$ & OA6 & Hanauma Bay & $5-7$ & $21^{\circ} 16^{\prime} 6.4^{\prime \prime}$ & $157^{\circ} 41^{\prime} 43.5^{\prime \prime}$ \\
\hline \multirow[t]{8}{*}{ Moloka'i } & 1 & MO1 & Pūko'o Nearshore & $1-6$ & $21^{\circ} 04^{\prime} 18.8^{\prime \prime}$ & $156^{\circ} 48^{\prime} 16.1^{\prime \prime}$ \\
\hline & 2 & $\mathrm{MO} 2$ & Pālā'au & 10 & $21^{\circ} 05^{\prime} 30.4^{\prime \prime}$ & $157^{\circ} 06^{\prime} 38.7^{\prime \prime}$ \\
\hline & $3^{* \dagger}$ & MO3 & Hale o Lono Reef & $9-12$ & $21^{\circ} 05^{\prime} 1.4^{\prime \prime}$ & $157^{\circ} 14^{\prime} 57.7^{\prime \prime}$ \\
\hline & 4 & MO4 & Pūko'o Offshore & $1-3$ & $21^{\circ} 03^{\prime} 55.8^{\prime \prime}$ & $156^{\circ} 47^{\prime} 38.4^{\prime \prime}$ \\
\hline & 5 & MO5 & Kamalō & $3-17$ & $21^{\circ} 02^{\prime} 40.6^{\prime \prime}$ & $156^{\circ} 54^{\prime} 1.1^{\prime \prime}$ \\
\hline & 6 & MO6 & Kamiloloa & $5-7$ & $21^{\circ} 04^{\prime} 18.2^{\prime \prime}$ & $157^{\circ} 00^{\prime} 9.7^{\prime \prime}$ \\
\hline & $7^{*+}$ & MO7 & Kaunakakai Reef & $7-10$ & $21^{\circ} 04^{\prime} 59.6^{\prime \prime}$ & $157^{\circ} 02^{\prime} 34.9^{\prime \prime}$ \\
\hline & 8 & MO8 & Hotel Moloka'i & $0.25-1$ & $21^{\circ} 04^{\prime} 28.7^{\prime \prime}$ & $156^{\circ} 59^{\prime} 48.7^{\prime \prime}$ \\
\hline \multirow[t]{9}{*}{ Maui } & 1 & MA1 & Kahekili & $1.5-5$ & $20^{\circ} 56^{\prime} 22.2^{\prime \prime}$ & $156^{\circ} 41^{\prime} 45.8^{\prime \prime}$ \\
\hline & 2 & MA1 & Olowalu & $2.5-3$ & $20^{\circ} 48^{\prime} 42.2^{\prime \prime}$ & $156^{\circ} 36^{\prime} 45.5^{\prime \prime}$ \\
\hline & 3 & MA1 & Papa'ula Point & $9-12$ & $20^{\circ} 55^{\prime} 39.3^{\prime \prime}$ & $156^{\circ} 25^{\prime} 44.9^{\prime \prime}$ \\
\hline & 4 & MA1 & Honolua Bay & $2.5-8.5$ & $21^{\circ} 01^{\prime} 8.9^{\prime \prime}$ & $156^{\circ} 38^{\prime} 33.2^{\prime \prime}$ \\
\hline & 5 & MO8 & Puamana & $3-3.5$ & $20^{\circ} 51^{\prime} 29.9^{\prime \prime}$ & $156^{\circ} 40^{\prime} 08.4^{\prime \prime}$ \\
\hline & 6 & MA1 & Māla Wharf & $3-9$ & $20^{\circ} 53^{\prime} 23.2^{\prime \prime}$ & $156^{\circ} 41^{\prime} 26.8^{\prime \prime}$ \\
\hline & $7 *+$ & MA1 & Mā‘alaea Reef & $2.5-5.5$ & $20^{\circ} 47^{\prime} 31.6^{\prime \prime}$ & $156^{\circ} 30^{\prime} 45.8^{\prime \prime}$ \\
\hline & 8 & MA1 & Kanahena Bay & $3-8$ & $20^{\circ} 38^{\prime} 5.3^{\prime \prime}$ & $156^{\circ} 29^{\prime} 57.6^{\prime \prime}$ \\
\hline & 9 & MA1 & Molokini Crater & $7-8.5$ & $20^{\circ} 37^{\prime} 14.9^{\prime \prime}$ & $156^{\circ} 26^{\prime} 30.8^{\prime \prime}$ \\
\hline \multirow[t]{10}{*}{ Hawai'i } & $1^{* \dagger}$ & HA1 & Kawaihae Reef & $4-9$ & $20^{\circ} 01^{\prime} 55.5^{\prime \prime}$ & $155^{\circ} 50^{\prime} 9.4^{\prime \prime}$ \\
\hline & 2 & HA2 & Puakō & $6-9$ & $19^{\circ} 58^{\prime} 23.2^{\prime \prime}$ & $155^{\circ} 51^{\prime} 04.4^{\prime \prime}$ \\
\hline & 3 & HA3 & 'Anaeho'omalu & $8.5-9$ & $19^{\circ} 57^{\prime} 2.2^{\prime \prime}$ & $155^{\circ} 52^{\prime} 08.4^{\prime \prime}$ \\
\hline & 4 & HA4 & Keawaiki & $12-14$ & $19^{\circ} 53^{\prime} 39.0^{\prime \prime}$ & $155^{\circ} 54^{\prime} 45.4^{\prime \prime}$ \\
\hline & 5 & HA5 & Kualani Point & $9.5-10.5$ & $19^{\circ} 33^{\prime} 05.7^{\prime \prime}$ & $155^{\circ} 57^{\prime} 54.2^{\prime \prime}$ \\
\hline & 6 & HA6 & Red Hill & $5.5-13.5$ & $19^{\circ} 30^{\prime} 28.8^{\prime \prime}$ & $155^{\circ} 57^{\prime} 19.5^{\prime \prime}$ \\
\hline & 7 & HA7 & North Keauhou & $6-6.5$ & $19^{\circ} 34^{\prime} 17.8^{\prime \prime}$ & $155^{\circ} 58^{\prime} 20.2^{\prime \prime}$ \\
\hline & 8 & HA8 & South Oneo Bay & $8-9$ & $19^{\circ} 38^{\prime} 04.8^{\prime \prime}$ & $156^{\circ} 01^{\prime} 33.4^{\prime \prime}$ \\
\hline & 9 & HA9 & Papawai Bay & $7-13.5$ & $19^{\circ} 39^{\prime} 00.6^{\prime \prime}$ & $156^{\circ} 01^{\prime} 33.4^{\prime \prime}$ \\
\hline & $10^{* \dagger}$ & HA10 & Leleiwi Bay & $6.5-10$ & $19^{\circ} 44^{\prime} 14.3^{\prime \prime}$ & $155^{\circ} 01^{\prime} 16.5^{\prime \prime}$ \\
\hline
\end{tabular}

*, Sites of sample collection and laboratory identification; ${ }^{\dagger}$, sites where rapid assessment surveys were conducted by two instead of three observers.

ing 18 nonindigenous and cryptogenic species occurred at only $1-3$ of the 41 sites surveyed.

The number of nonindigenous and cryptogenic species recorded by rapid assessment at each site surveyed is also shown in Figure 6. A maximum of six nonindigenous and cryptogenic species occurred at one site each at Port Allen Harbor Reef, Kaua'i (KA5);
Māla Wharf, Maui (MA6); and Kāne'ohe Bay, $\mathrm{O}^{\prime}$ ahu (OA2). Five nonindigenous and cryptogenic species occurred at the other Kāne'ohe Bay site (OA1); the Marriott Hotel Reef, Kaua'i (MA1); and at the Puakō and Red Hill sites on Hawai'i (HA2 and HA6). No nonindigenous and cryptogenic species were detected at the Kamiloloa, Moloka'i (MO6); 
TABLE 2

Description of Characteristics for Each Category of Index Values

\begin{tabular}{lcl}
\hline \hline Index & Value & \\
\hline Artificial substrate & 1 & No modification of shoreline or artificial structure in water \\
& 2 & $<25 \%$ of shoreline hardening, no structure in the water \\
& 3 & $26-50 \%$ shoreline hardening or structures in the water \\
Urbanization & 4 & $51-75 \%$ shoreline hardening \\
& 5 & Shoreline $>75 \%$ modified or hardened \\
& 1 & Remote: no habitation or shoreline development in view \\
& 2 & Rural: 1 to 5 houses or buildings, no shoreline development \\
Ocean restriction & 3 & Residential: 6 to 30 houses or buildings, some development at or near shoreline \\
& 4 & Urbanized: $>30$ houses or buildings, high shoreline development \\
& 5 & Industrialized, commercial shoreline usage and development \\
& 2 & Open ocean \\
Reef condition & 3 & Semiexposed coastline \\
& 4 & Embayment \\
& 5 & Semienclosed harbor \\
& 1 & Excellent: coral cover $>50 \%$ \\
& 2 & Good: coral cover 26-50\%, macroalgae rare \\
& 3 & Fair: coral cover 11-25\%, some algae present \\
& 4 & Poor: coral cover 6-10\%, algae common to abundant \\
& 5 & Highly degraded: coral cover 0-5\%, high sedimentation \\
\hline
\end{tabular}
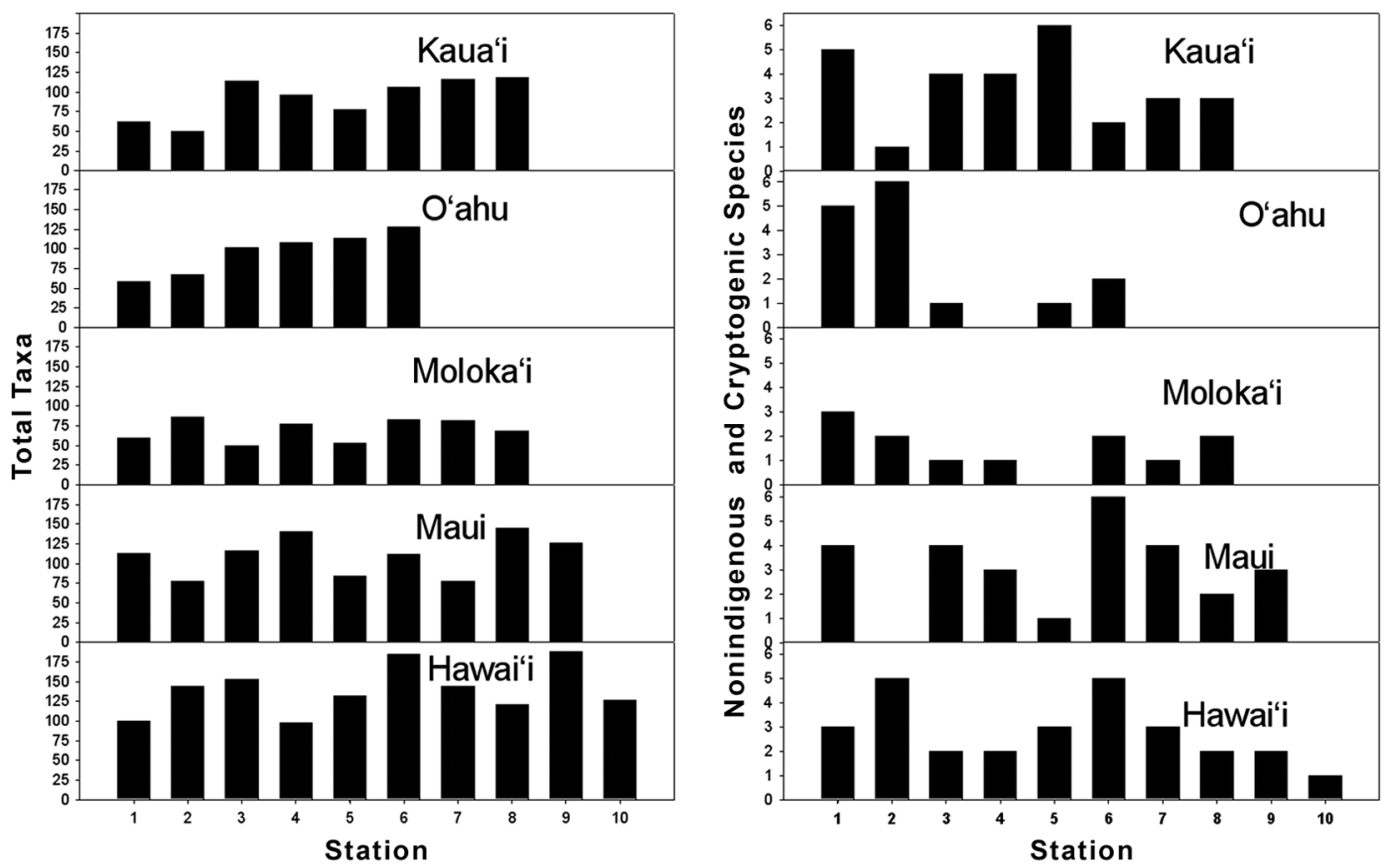

FiguRE 6. Distributions of numbers of total taxa and nonindigenous and cryptogenic species determined by rapid assessment. 
TABLE 3

Introduced and Cryptogenic Species Recorded by Rapid Assessments

\begin{tabular}{|c|c|c|c|c|c|c|c|c|c|c|c|c|}
\hline \multirow[b]{2}{*}{ Island } & \multirow[b]{2}{*}{ Species } & \multirow[b]{2}{*}{ Status } & \multicolumn{10}{|c|}{ Site } \\
\hline & & & 1 & 2 & 3 & 4 & 5 & 6 & 7 & 8 & 9 & 10 \\
\hline \multirow[t]{13}{*}{ Kaua'i } & Mycale cf. armata Thiele, 1903 & Introduced & $\mathrm{x}$ & & & & & & & & & \\
\hline & Gelloides cf. fibrosa (Wilson, 1925) & Introduced & $\mathrm{x}$ & & & & & & & & & \\
\hline & Dynamena crisoides Lamaroux, 1824 & Cryptogenic & & & & & $\mathrm{x}$ & & & & & \\
\hline & Pennaria disticha (Goldfuss, 1820) & Introduced & $\mathrm{x}$ & & $\mathrm{x}$ & & $\mathrm{x}$ & & & & & \\
\hline & Chaetopterus sp. & Cryptogenic & $\mathrm{x}$ & & & & & & & & & \\
\hline & Sabellastarte spectabilis (Grube, 1878) & Introduced & $\mathrm{x}$ & & & & $\mathrm{x}$ & & & & & \\
\hline & Salmacina dysteri (Huxley, 1855) & Introduced & & & & $\mathrm{x}$ & & & & & & \\
\hline & Hipponix australis (Lamarck, 1819) & Cryptogenic & & & $\mathrm{x}$ & $\mathrm{x}$ & & & $\mathrm{x}$ & $\mathrm{x}$ & & \\
\hline & Schizoporella cf. errata (Waters, 1878) & Introduced & & & & & & $\mathrm{x}$ & & & & \\
\hline & Didemnum cf. candidum Savigny, 1816 & Introduced & & $\mathrm{x}$ & $\mathrm{x}$ & $\mathrm{x}$ & $\mathrm{x}$ & $\mathrm{x}$ & $\mathrm{x}$ & $\mathrm{x}$ & & \\
\hline & Lutjanus kasmira (Forsskål, 1775) & Introduced & & & $\mathrm{x}$ & & $\mathrm{x}$ & & $\mathrm{x}$ & & & \\
\hline & Cephalopholis argus Bloch \& Schneider, 1801 & Introduced & & & & $\mathrm{x}$ & $\mathrm{x}$ & & & $\mathrm{x}$ & & \\
\hline & Total nonindigenous and cryptogenic species & & 5 & 1 & 4 & 4 & 6 & 2 & 3 & 3 & & \\
\hline \multirow[t]{12}{*}{ O‘ahu } & Acanthophora spicifera (Vahl) Børgesen & Introduced & & & $\mathrm{x}$ & & & & & & & \\
\hline & Kappaphycus alvarezii (Doty) Doty & Introduced & $\mathrm{x}$ & $\mathrm{x}$ & & & & & & & & \\
\hline & Mycale cf. armata Thiele, 1903 & Introduced & $\mathrm{x}$ & $\mathrm{x}$ & & & & & & & & \\
\hline & Sigmadocia caerulea Hechtel, 1965 & Introduced & & & & & $\mathrm{x}$ & & & & & \\
\hline & Sabellastarte cf. spectabilis (Grube, 1878) & Introduced & $\mathrm{x}$ & $\mathrm{x}$ & & & & & & & & \\
\hline & Anomia nobilis Reeve, 1859 & Introduced & $\mathrm{x}$ & $\mathrm{x}$ & & & & & & & & \\
\hline & Phallusia nigra Savigny, 1816 & Introduced & & $\mathrm{x}$ & & & & & & & & \\
\hline & Didemnum cf. candidum Savigny, 1816 & Cryptogenic & $\mathrm{x}$ & & & & & & & & & \\
\hline & Lutjanus kasmira (Forsskål, 1775) & Introduced & & & & & & $\mathrm{x}$ & & & & \\
\hline & Centropyge loricula (Günther, 1873) & Cryptogenic & & $\mathrm{x}$ & & & & & & & & \\
\hline & Cephalopholis argus Bloch \& Schneider, 1801 & Introduced & & & & & & $\mathrm{x}$ & & & & \\
\hline & Total nonindigenous and cryptogenic species & & 5 & 6 & 1 & 0 & 1 & 2 & & & & \\
\hline \multirow[t]{6}{*}{ Moloka'i } & Acanthophora spicifera (Vahl) Børgesen & Introduced & $\mathrm{x}$ & & & $\mathrm{x}$ & & & & $\mathrm{x}$ & & \\
\hline & Cassiopea cf. andromeda Light, 1914 & Introduced & $\mathrm{x}$ & & & & & & & & & \\
\hline & Hipponix australis (Lamarck, 1819) & Cryptogenic & & & & & & $\mathrm{x}$ & & & & \\
\hline & Didemnum cf. candidum Savigny, 1816 & Introduced & $\mathrm{x}$ & $\mathrm{x}$ & & & & $\mathrm{x}$ & & $\mathrm{x}$ & & \\
\hline & Cephalopholis argus Bloch \& Schneider, 1801 & Introduced & & $\mathrm{x}$ & $\mathrm{x}$ & & & & $\mathrm{x}$ & & & \\
\hline & Total nonindigenous and cryptogenic species & & 3 & 2 & 1 & 1 & 0 & 2 & 1 & 2 & & \\
\hline \multirow[t]{13}{*}{ Maui } & Hypnea musciformis (Wulfen) J. V. Lamour & Introduced & & & & & & & $\mathrm{x}$ & & & \\
\hline & Acanthophora spicifera (Vahl) Børgesen & Introduced & $\mathrm{x}$ & & $\mathrm{x}$ & & & & $\mathrm{x}$ & & & \\
\hline & Hipponix australis (Lamarck, 1819) & Cryptogenic & $\mathrm{x}$ & & & $\mathrm{x}$ & $\mathrm{x}$ & $\mathrm{x}$ & & & & \\
\hline & Mycale cf. armata Thiele, 1903 & Introduced & & & & & & $\mathrm{x}$ & $\mathrm{x}$ & & & \\
\hline & Pennaria disticha (Goldfuss, 1820) & Introduced & & & & & & $\mathrm{x}$ & & & & \\
\hline & Sarcothelia, undescribed sp. & Cryptogenic & & & $\mathrm{x}$ & & & & & & & \\
\hline & Carijoa riisei (Duchassaing \& Michelotti, 1860) & Introduced & & & & & & $\mathrm{x}$ & & & & \\
\hline & Crassostrea sp. & Introduced & & & & & & & $\mathrm{x}$ & & & \\
\hline & Didemnum cf. candidum Savigny, 1816 & Introduced? & $\mathrm{x}$ & & $\mathrm{x}$ & & & & & & & \\
\hline & Cephalopholis argus Bloch \& Schneider, 1801 & Introduced & $\mathrm{x}$ & & $\mathrm{x}$ & $\mathrm{x}$ & & $\mathrm{x}$ & & $\mathrm{x}$ & $\mathrm{x}$ & \\
\hline & Lutjanus kasmira (Quoy \& Gaimard, 1825) & Introduced & & & & $\mathrm{x}$ & & & & & $\mathrm{x}$ & \\
\hline & Lutjanus fulvus (Forster, 1801) & Introduced & & & & & & $\mathrm{x}$ & & & $\mathrm{x}$ & \\
\hline & Total nonindigenous and cryptogenic species & & 4 & 0 & 4 & 3 & 1 & 6 & 4 & 1 & 3 & \\
\hline \multirow[t]{9}{*}{ Hawai' $\mathrm{i}$} & Pennaria disticha (Goldfuss, 1820) & Introduced & $\mathrm{x}$ & & & & & & & & & \\
\hline & Plumularia floridana (Nutting, 1905) & Cryptogenic & $\mathrm{x}$ & & & & & $\mathrm{x}$ & & & & \\
\hline & Plumularia strictocarpa Pictect, 1893 & Cryptogenic & & & & & & $\mathrm{x}$ & & & & \\
\hline & Sabellastarte spectabilis (Grube, 1878) & Introduced & & $\mathrm{x}$ & & & & & & & & \\
\hline & Hipponix australis Lamarck, 1819 & Cryptogenic & & $\mathrm{x}$ & $\mathrm{x}$ & $\mathrm{x}$ & $\mathrm{x}$ & $\mathrm{x}$ & $\mathrm{x}$ & $\mathrm{x}$ & $\mathrm{x}$ & \\
\hline & Lutjanus fulvus (Forster, 1801) & Introduced & & $\mathrm{x}$ & & & & & & & & \\
\hline & Lutjanus kasmira (Forsskål, 1775) & Introduced & & $\mathrm{x}$ & & & $\mathrm{x}$ & $\mathrm{x}$ & $\mathrm{x}$ & & & \\
\hline & Cephalopholis argus Bloch \& Schneider, 1801 & Introduced & $\mathrm{x}$ & $\mathrm{x}$ & $\mathrm{x}$ & $\mathrm{x}$ & $\mathrm{x}$ & $\mathrm{x}$ & $\mathrm{x}$ & $\mathrm{x}$ & $\mathrm{x}$ & $\mathrm{x}$ \\
\hline & Total nonindigenous and cryptogenic species & & 3 & 5 & 2 & 2 & 3 & 5 & 3 & 2 & 2 & 1 \\
\hline
\end{tabular}




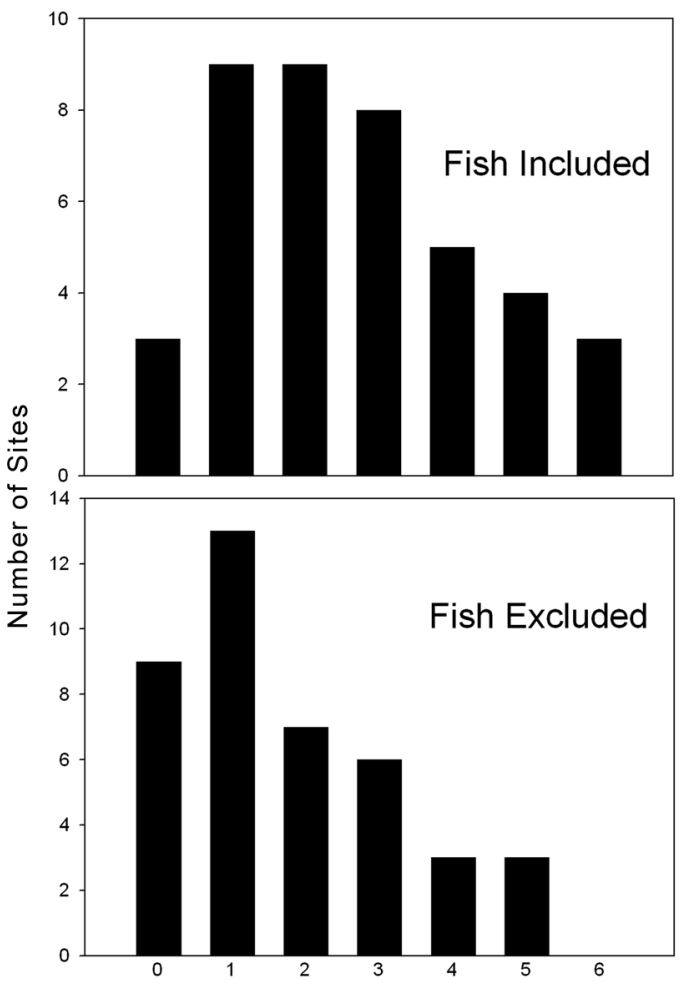

Number of N/CS

FigURE 7. Histograms of numbers of nonindigenous and cryptogenic species (N/CS) at all sites with fish data included and excluded.

Olowalu, Maui (MA2); or Kahe, O'ahu (OA4) sites. Histograms of nonindigenous and cryptogenic species occurrence values (Figure 7) indicate that 21 of the 41 sites $(51 \%)$ had fewer than three nonindigenous and cryptogenic species. Many of these were sites where only introduced fishes (Lutjanus kasmira, Lutjanus fulvus (Forster), and Cephalopholis argus) occurred. These three species were intentionally introduced in the 1950s (Brock 1960, Randall 1987) and are widespread throughout the main Hawaiian Islands (Randall and Kanayama 1972, Maciolek 1984). When these three species are excluded from the results (Figure 7), 22 of the 41 sites $(54 \%)$ had fewer than two nonindigenous and cryptogenic species.

\section{Evaluation of Rapid Assessment Technique}

The numbers of total taxa, total nonindigenous and cryptogenic species, and total native species richness recorded at the seven sites where samples were collected at Kaua'i, Moloka'i, Maui, and Hawai'i are shown in Table 4 for rapid assessments and for total taxa that were identified in the laboratory. Six of the 54 species were new reports for the Hawaiian Islands designated cryptogenic, and 20 more were not previously reported outside $\mathrm{O}^{\prime} \mathrm{ahu}$ in published or unpublished literature or in Bishop Museum collections. Numbers of total taxa, native species richness, and nonindigenous and cryptogenic species were many times greater for the laboratory-identified samples than for the rapid assessment observations, as was anticipated because all minute and cryptic species could be identified in the laboratory. However, there was good agreement for each site between both methods for the ratios of nonindigenous and cryptogenic species to native species richness. Values for nonindigenous and cryptogenic species ranged from 2.4 to $9.1 \%$ for the collected samples and 1.0 to $11.1 \%$ for the rapid assessments (Table 4), with a significant correlation between the values from each site (Figure 8). Similar agreement in results was found in a previous study of 25 stations in Kāne'ohe Bay where both on-site observations and laboratory identifications showed $19 \%$ of the total identified organisms to be composed of nonindigenous and cryptogenic species (Coles et al. 2002a; S.L.C., unpubl. data).

As a further determination of the sensitivity and consistency of the rapid assessment method for detecting introductions, we calculated, for the seven stations where samples were collected, the ratios of numbers of species recorded from rapid assessments (RA) to numbers of taxa identified from laboratory identifications (LID) for both introduced/ cryptogenic (N/CS $\left.\mathrm{RA}: \mathrm{N} / \mathrm{CS}_{\mathrm{LID}}\right)$ and native species richness $\left(\mathrm{NSR}_{\mathrm{RA}}: \mathrm{NSR}_{\mathrm{LID}}\right)$ data $(\mathrm{Ta}-$ ble 4). These ratios would be constant if nonindigenous and cryptogenic species determined from rapid assessments were a consistent percentage of the total biota as 


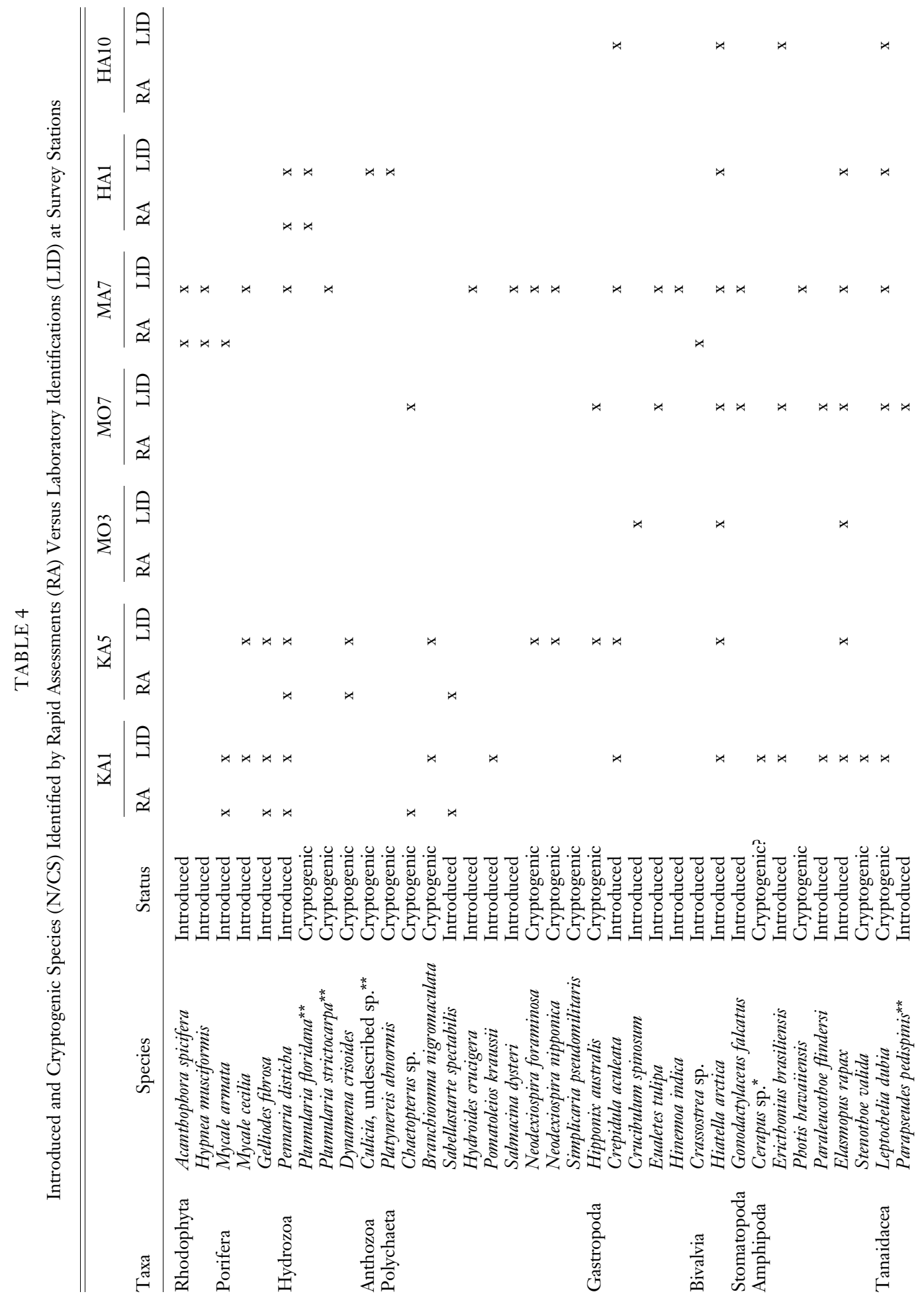




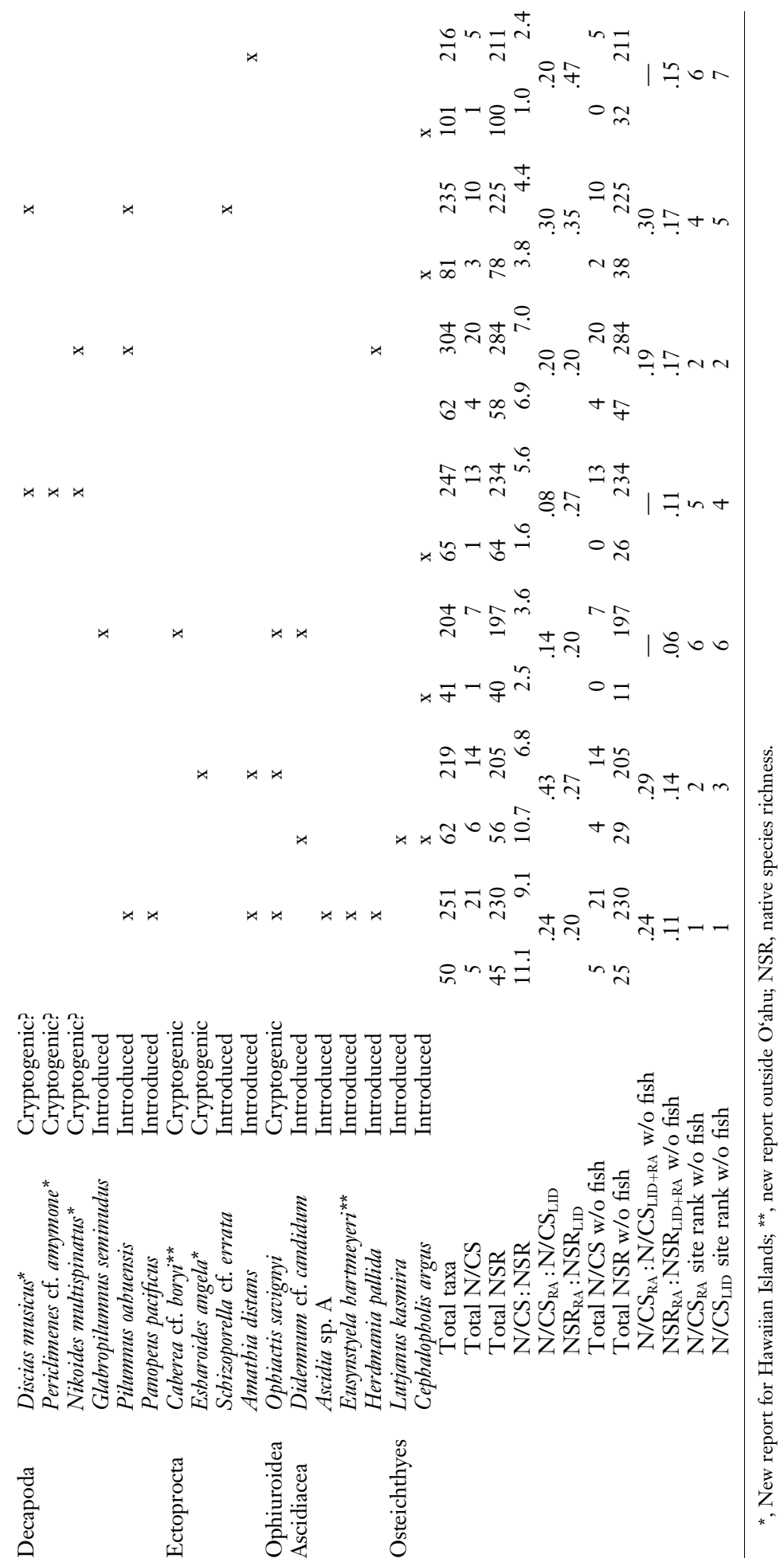




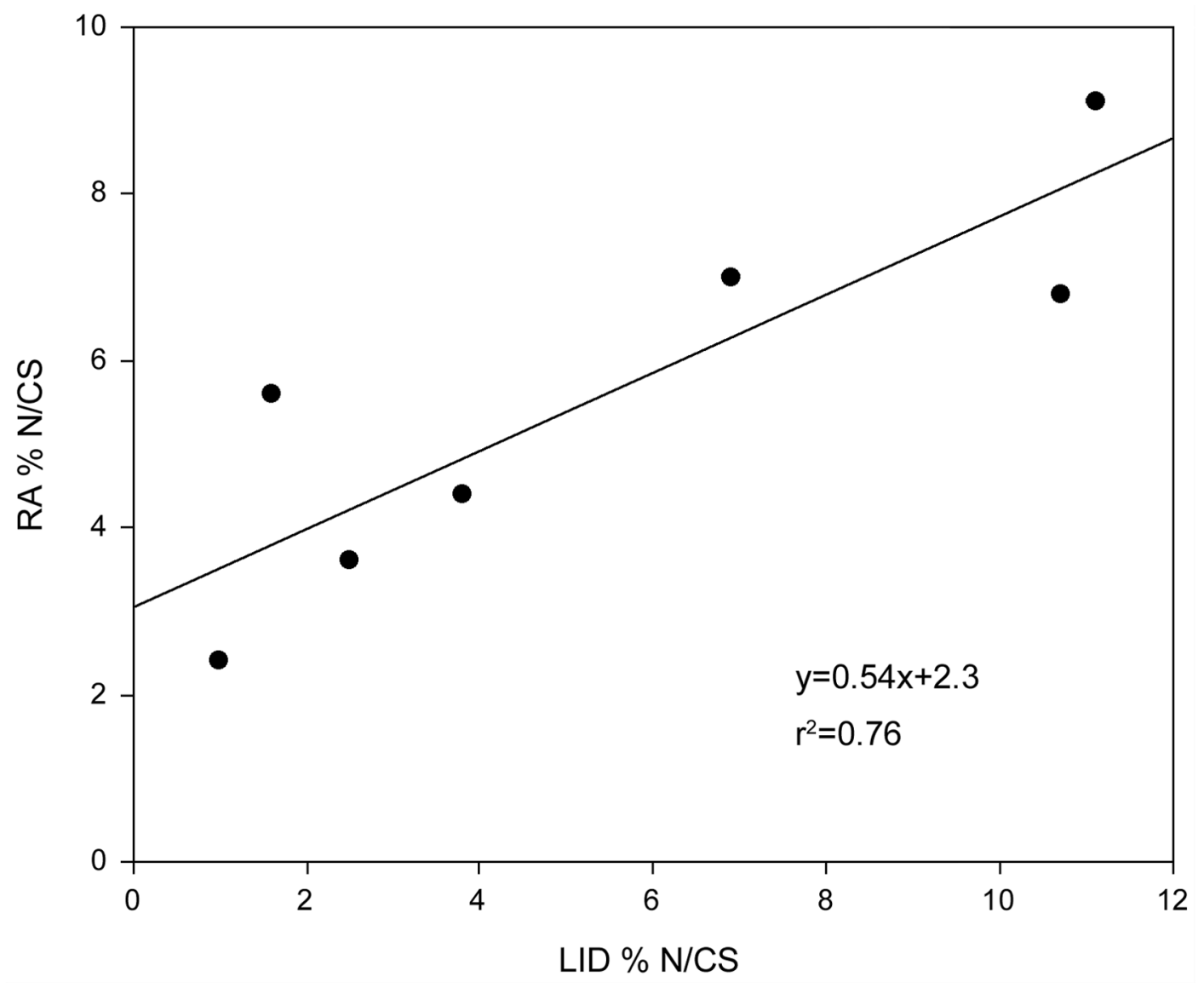

FIGURE 8. Comparison of percentage nonindigenous and cryptogenic species (N/CS) recorded on rapid assessment (RA) surveys with percentage nonindigenous and cryptogenic species from laboratory identifications (LID) + rapid assessments from the same sites.

determined from laboratory identifications. However, the scatter of these ratios (Figure $9 a$ ) indicates that rapid assessments detected an inconsistent percentage of the biota to be nonindigenous and cryptogenic species. Further, most of the points fall below the line representing a $1: 1$ ratio, suggesting that rapid assessments tended to underestimate nonindigenous and cryptogenic species relative to native species richness.

Inspection of the data suggested that much of this inconsistency was due to inclusion of fish species, because the introduced fishes usually occurred at open-ocean reef sites where few to no other introduced species oc- curred and where fishes were a dominant portion of the total identified biota. Calculating and plotting the same ratios for the data with fish excluded reduced the number of sites to the four where only nonfish nonindigenous and cryptogenic species occurred (Table 4). These show a more consistent pattern (Figure $9 b$ ), with ratios ranging from 0.11 to 0.30 and all points plotting above a line representing equivalent ratios for nonindigenous and cryptogenic species ${ }_{\mathrm{RA}}$ : nonindigenous and cryptogenic species LID $_{\text {and }}$ native species richness $_{\mathrm{RA}}$ : native species richness ${ }_{\text {LID. }}$. This suggests that, in contrast to the pattern shown in Figure $9 a$, the rapid assessment method 

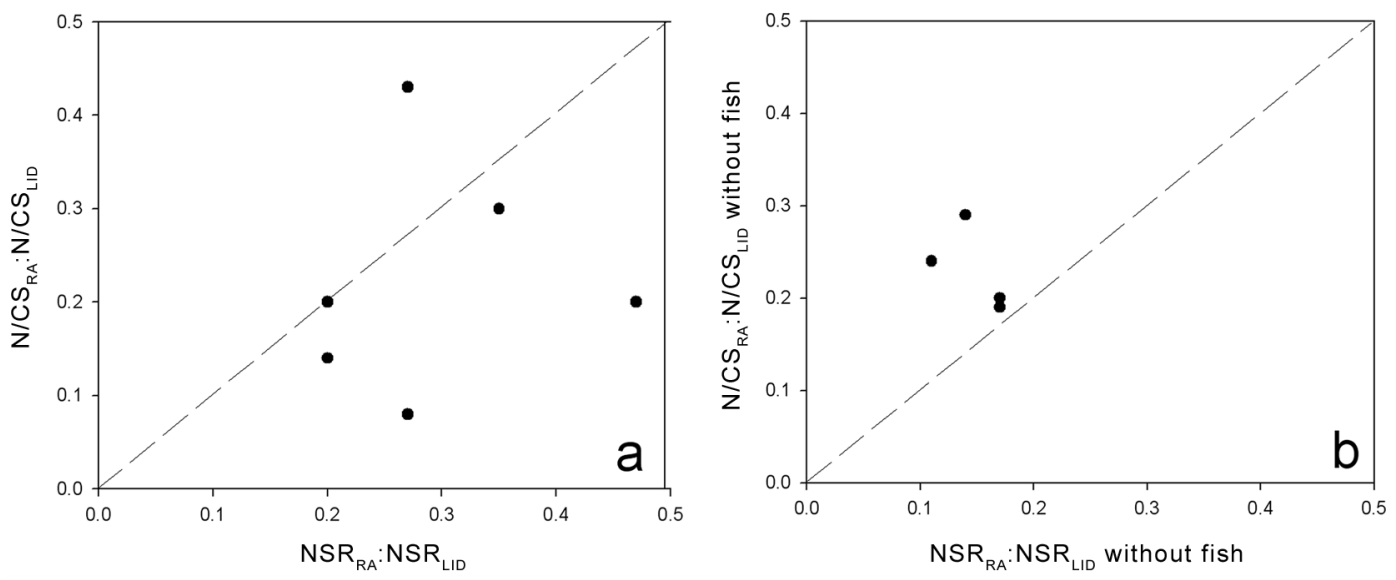

FIGURE 9. Ratios of native species richness (NSR) recorded on rapid assessment (RA) surveys and from laboratory identifications (LID) + rapid assessments from the same sites $\left(\mathrm{NSR}_{\mathrm{RA}}: \mathrm{NSR}_{\mathrm{LID}+\mathrm{RA}}\right)$ plotted against nonindigenous and cryptogenic species $\left(\mathrm{N} / \mathrm{CS}_{\mathrm{RA}}: \mathrm{N} / \mathrm{CS}_{\mathrm{LID}+\mathrm{RA}}\right)$ for $a$, all sample collection sites; $b$, same data with fish excluded.
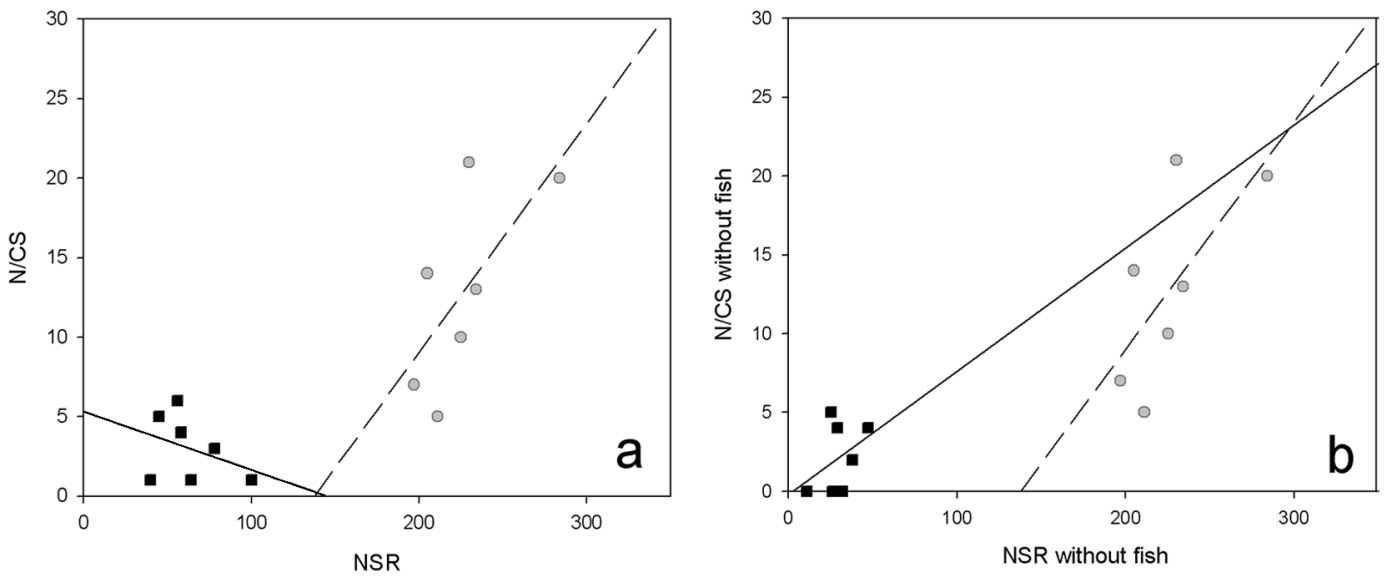

FIGURE 10. Regressions of nonindigenous and cryptogenic species (N/CS) on native species richness (NSR) for taxa identified by rapid assessment (black squares, solid lines) and by laboratory identification + rapid assessment (gray circles, dashed lines) for $a$, all sample collection sites; $b$, same data with fish excluded.

with fish excluded detected slightly higher percentages of nonindigenous and cryptogenic species relative to native species than did laboratory identifications.

A second analysis showed a similar inconsistency between rapid assessment and laboratory identification results that is reduced when the fish data are excluded. A plot of numbers of nonindigenous and cryptogenic species against native species richness for the full data set (Figure 10a) shows a positive relationship for the laboratory identification results but a negative relationship for the rapid assessment results, suggesting that rapid assessments underestimated nonindigenous and cryptogenic species at the most species-rich sites. Excluding fish data from both nonindigenous and cryptogenic species and native species richness results rectified some of this inconsistency (Figure 10b). The resulting 
regression for rapid assessment results is positive and more comparable with the laboratory identification results. Finally, both rapid assessments and the laboratory identifications showed similar rank orders of the stations for number of nonfish nonindigenous and cryptogenic species reports (Table 4), with Stations KA1 and MA7 ranking highest and Stations MO3 and HA10 ranking lowest for both rapid assessments and laboratory identifications.

\section{Variables Influencing Introductions}

Given the above results, fishes were excluded from the following analysis. Also, because the third field team member did not make rapid assessment observations at all of the sites, the rapid assessment data from that observer were not included in the analysis of relationships between numbers of nonindigenous and cryptogenic species and environmental variables (Table 2) or native species richness to compare data based on consistent effort throughout the study. These exclusions resulted in the total number of taxa used in regression analysis being reduced to 208 from the 486 reported by all three observers. However, algae and invertebrate nonindigenous and cryptogenic species (Table 3) were reduced by only three species: cryptogenic Hipponix australis that occurred at 20 sites, and introduced polychaete Salmacina dysteri (Huxley) and introduced bryozoan Schizoporella cf. errata (Waters), each of which occurred at only one site.

The sites surveyed encompassed a wide range of environmental conditions and proximity to anthropogenic influences on each island (Table 5). The stations ranged from $100 \mathrm{~m}$ to $4.6 \mathrm{~km}$ from the shoreline and from $300 \mathrm{~m}$ to $26 \mathrm{~km}$ from the nearest stream mouth. Distances from harbors ranged from $300 \mathrm{~m}$ to $71 \mathrm{~km}$ and those from boatlaunching ramps from $200 \mathrm{~m}$ to $27 \mathrm{~km}$. Most of the sites (18-25) were in the lowest index category (1) for artificial substrata, reef condition, and ocean restriction (i.e., most sites were in the category considered least likely to promote nonindigenous and cryptogenic species for these indexes). Over half of the values were in categories 3 to 5 for the urbanization index (more than six houses or buildings visible on the shoreline).

Values for ranked variables; distances from harbor piers, boat-launching areas, shorelines, and streams; and numbers of native taxa excluding fishes (Table 5) were used as potential predictor variables in best subsets regression analysis (Minitab Release 14.1, Minitab, State College, Pennsylvania) to identify the best-fitting regression model explaining numbers of nonindigenous and cryptogenic species. The initial model with the most explanatory power (adjusted $\left.r^{2}=0.52\right)$ included the three predictor variables ocean isolation, ramp distance, and native species richness in order of variance explained. These three predictor variables and all of their possible two-way interactions were then used in a best subsets regression analysis to identify the most predictive and logical model (i.e., if an interaction term was included in the model, the variables used to calculate the interaction term were also included). This best regression model accounted for $67.2 \%$ of the variance explained and included as significant factors ocean isolation, native species richness, and their interaction (Table 6). Their relationship with numbers of nonindigenous and cryptogenic species is illustrated graphically in Figure 11. Most $(45 \%)$ of the variance in nonindigenous and cryptogenic species was explained by a highly significant positive relationship $(P<.001)$ between numbers of nonindigenous and cryptogenic species and isolation from open-ocean conditions (i.e., nonindigenous and cryptogenic species increased significantly going from open coastlines to semienclosed locations). This effect was modified by a highly significant $(P<.001)$ negative isolation-native species richness interaction term where nonindigenous and cryptogenic species decreased as native species richness and isolation increased. Examining numbers of nonindigenous and cryptogenic species and native species richness in the four categories of isolation index 
TABLE 5

Matrix of Predictor Variables Used in Best Subsets Regression Analysis at 41 Reef Sites

\begin{tabular}{|c|c|c|c|c|c|c|c|c|c|c|c|}
\hline Island & Station & $\begin{array}{c}\text { Harbor } \\
\text { Distance } \\
(\mathrm{km})\end{array}$ & $\begin{array}{l}\text { Ramp } \\
\text { Distance } \\
(\mathrm{km})\end{array}$ & $\begin{array}{l}\text { Shore } \\
\text { Distance } \\
(\mathrm{km})\end{array}$ & $\begin{array}{l}\text { Stream } \\
\text { Distance } \\
(\mathrm{km})\end{array}$ & $\begin{array}{c}\text { Artificial } \\
\text { Substrate } \\
\text { Index }\end{array}$ & $\begin{array}{c}\text { Reef } \\
\text { Health } \\
\text { Index }\end{array}$ & $\begin{array}{l}\text { Urban- } \\
\text { ization } \\
\text { Index }\end{array}$ & $\begin{array}{l}\text { Isolation } \\
\text { Index }\end{array}$ & NSR & $\begin{array}{l}\text { N/CS } \\
\text { (w/o } \\
\text { fish) }\end{array}$ \\
\hline \multirow[t]{8}{*}{ Kaua'i } & 1 & 1.2 & 1.5 & 0.3 & 0.3 & 4 & 3 & 3 & 3 & 45 & 5 \\
\hline & 2 & 12.7 & 1.7 & 0.1 & 1.1 & 2 & 4 & 4 & 2 & 32 & 0 \\
\hline & 3 & 13.1 & 2.1 & 0.2 & 0.5 & 1 & 2 & 3 & 1 & 77 & 2 \\
\hline & 4 & 13.4 & 1.9 & 0.2 & 0.7 & 1 & 2 & 3 & 1 & 56 & 0 \\
\hline & 5 & 0.6 & 0.7 & 0.4 & 0.7 & 4 & 5 & 2 & 4 & 58 & 3 \\
\hline & 6 & 8.5 & 8.6 & 0.6 & 0.7 & 2 & 5 & 2 & 1 & 59 & 0 \\
\hline & 7 & 3.9 & 4.0 & 0.1 & 3.1 & 1 & 3 & 1 & 1 & 65 & 0 \\
\hline & 8 & 11.9 & 0.6 & 0.1 & 2.0 & 3 & 3 & 4 & 1 & 74 & 0 \\
\hline \multirow[t]{6}{*}{$\mathrm{O}^{\prime} \mathrm{ahu}$} & 1 & 4.4 & 4.5 & 1.1 & 1.4 & 1 & 3 & 4 & 3 & 46 & 5 \\
\hline & 2 & 0.3 & 0.5 & 0.3 & 0.7 & 2 & 4 & 3 & 3 & 56 & 5 \\
\hline & 3 & 6.2 & 6.1 & 0.1 & 0.3 & 1 & 4 & 3 & 1 & 81 & 1 \\
\hline & 4 & 3.4 & 3.3 & 0.1 & 3.2 & 3 & 3 & 3 & 1 & 87 & 0 \\
\hline & 5 & 7.5 & 7.6 & 0.1 & 0.6 & 1 & 2 & 3 & 1 & 93 & 1 \\
\hline & 6 & 5.4 & 5.6 & 0.1 & 5.8 & 1 & 2 & 2 & 3 & 105 & 0 \\
\hline \multirow[t]{8}{*}{ Moloka'i } & 1 & 0.3 & 0.3 & 0.1 & 0.4 & 3 & 4 & 3 & 2 & 37 & 2 \\
\hline & 2 & 8.4 & 8.4 & 1.4 & 8.3 & 1 & 1 & 2 & 1 & 60 & 0 \\
\hline & 3 & 0.6 & 22.7 & 0.5 & 23.1 & 3 & 1 & 3 & 1 & 40 & 0 \\
\hline & 4 & 1.2 & 26.9 & 1.0 & 1.3 & 3 & 3 & 3 & 1 & 54 & 1 \\
\hline & 5 & 12.0 & 15.3 & 1.5 & 1.7 & 2 & 1 & 4 & 1 & 33 & 0 \\
\hline & 6 & 3.6 & 5.3 & 1.6 & 5.3 & 3 & 1 & 4 & 1 & 43 & 0 \\
\hline & 7 & 1.1 & 1.5 & 1.4 & 1.5 & 3 & 1 & 3 & 1 & 64 & 0 \\
\hline & 8 & 4.1 & 4.3 & 0.6 & 4.6 & 3 & 4 & 4 & 1 & 56 & 1 \\
\hline \multirow[t]{9}{*}{ Maui } & 1 & 32.3 & 6.0 & 0.1 & 1.6 & 1 & 2 & 4 & 2 & 71 & 2 \\
\hline & 2 & 12.4 & 12.5 & 0.2 & 1.9 & 2 & 3 & 2 & 2 & 52 & 0 \\
\hline & 3 & 6.2 & 6.3 & 1.6 & 2.7 & 1 & 1 & 1 & 1 & 75 & 2 \\
\hline & 4 & 27.4 & 17.7 & 0.1 & 0.4 & 1 & 1 & 1 & 3 & 96 & 0 \\
\hline & 5 & 21.1 & 5.3 & 0.1 & 0.5 & 1 & 3 & 2 & 1 & 46 & 0 \\
\hline & 6 & 25.7 & 0.2 & 0.2 & 0.3 & 5 & 2 & 3 & 4 & 73 & 4 \\
\hline & 7 & 0.3 & 0.4 & 0.3 & 10.4 & 3 & 4 & 4 & 3 & 58 & 4 \\
\hline & 8 & 20.6 & 20.8 & 0.1 & 26.0 & 1 & 1 & 2 & 2 & 87 & 0 \\
\hline & 9 & 17.7 & 17.9 & 4.6 & 23.0 & 1 & 1 & 1 & 1 & 83 & 0 \\
\hline \multirow[t]{10}{*}{ Hawai'i } & 1 & 1.8 & 1.5 & 0.4 & 1.8 & 4 & 1 & 2 & 1 & 78 & 2 \\
\hline & 2 & 8.2 & 7.9 & 0.2 & 5.4 & 2 & 1 & 3 & 1 & 89 & 1 \\
\hline & 3 & 10.9 & 10.6 & 0.3 & 8.0 & 1 & 1 & 2 & 1 & 85 & 0 \\
\hline & 4 & 18.8 & 18.5 & 0.5 & 16.4 & 1 & 1 & 2 & 1 & 58 & 0 \\
\hline & 5 & 68.5 & 16.2 & 0.2 & 9.2 & 1 & 1 & 1 & 1 & 75 & 0 \\
\hline & 6 & 71.0 & 18.9 & 0.1 & 12.0 & 1 & 1 & 2 & 1 & 106 & 0 \\
\hline & 7 & 64.3 & 14.1 & 0.2 & 6.8 & 1 & 1 & 4 & 1 & 83 & 0 \\
\hline & 8 & 57.4 & 7.4 & 0.2 & 1.2 & 2 & 1 & 5 & 2 & 74 & 0 \\
\hline & 9 & 53.5 & 3.9 & 0.1 & 4.7 & 1 & 1 & 1 & 1 & 111 & 0 \\
\hline & 10 & 8.8 & 8.9 & 0.1 & 8.0 & 1 & 2 & 3 & 1 & 100 & 0 \\
\hline
\end{tabular}

NSR, native species richness; N/CS, nonindigenous and cryptogenic species.

(Figure 12) showed no significant relationship in open-ocean or semiexposed coastlines, but nonindigenous and cryptogenic species decreased significantly with native species richness in the combined embayment and semienclosed harbor environments.

\section{DISCUSSION}

Our results agree with previous findings in the Hawaiian Islands, Johnston Atoll, American Samoa, and Guam indicating that relatively few introduced marine invertebrates 
TABLE 6

Relationships Determined between Numbers of Nonindigenous and Cryptogenic Species (N/CS) and Predictor Variables by Best Subsets Linear Regression Analysis

\begin{tabular}{lccccc}
\hline \hline $\begin{array}{l}\text { Potential Determining } \\
\text { Factor }\end{array}$ & Type & Range & $\begin{array}{c}\text { Expected } \\
\text { N/CS Effect }\end{array}$ & Result & $\begin{array}{c}\text { \% Variance } \\
\text { Explained }\end{array}$ \\
\hline Ocean isolation & Estimated & $1-5$ & + & $+P<0.001$ & 45.3 \\
Species richness (NSR) & Measured & $42-138$ & - & $+P<0.030$ & 8.0 \\
Isolation-NSR interaction & & & & $-P<0.001$ & 13.9 \\
Full model & & & & $+P<0.001$ & 67.2 \\
\hline
\end{tabular}

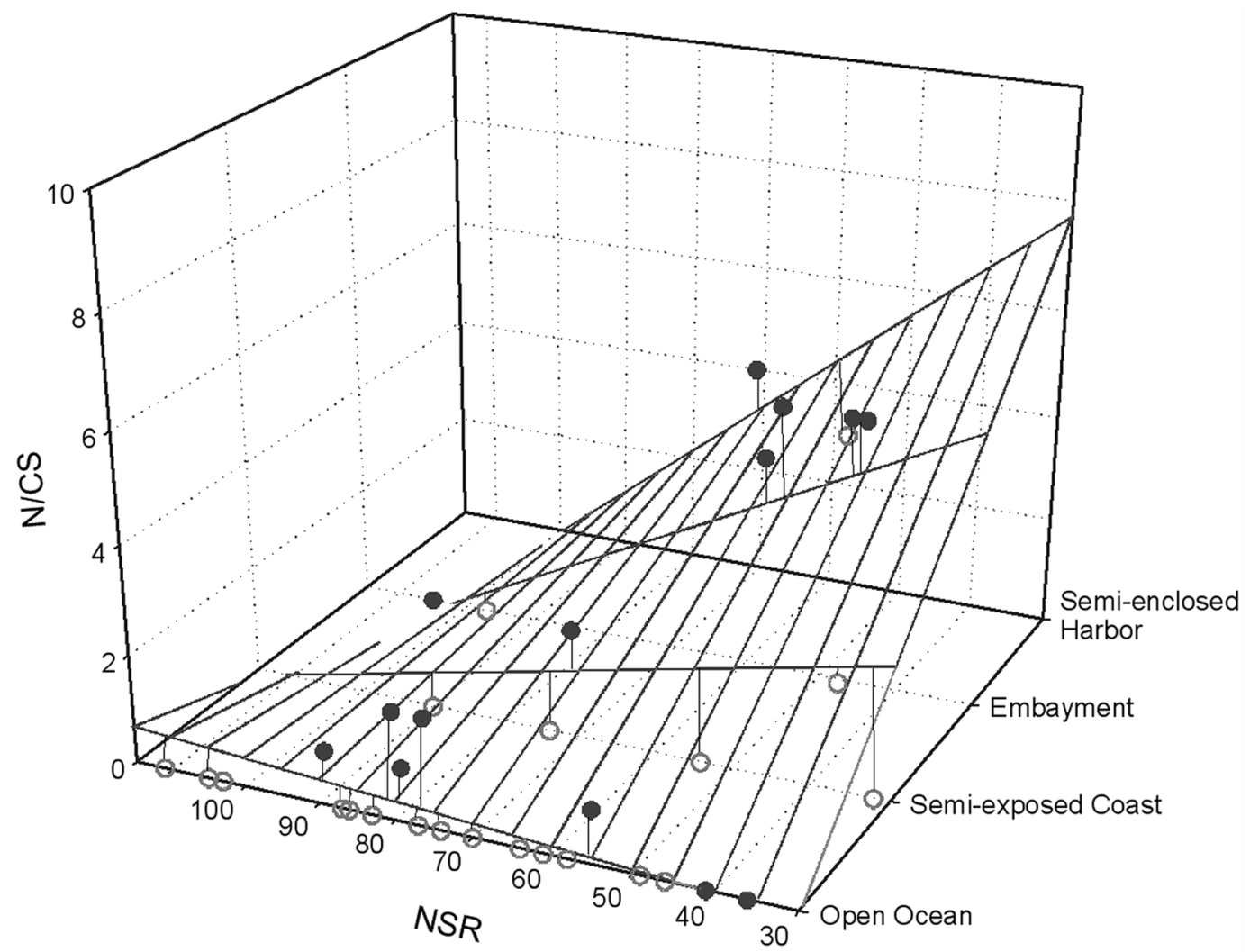

Figure 11. Three-dimensional response surface illustrating relationships between numbers of nonindigenous and cryptogenic species (N/CS), ocean exposure of the sites surveyed, and native species richness (NSR, fish excluded). $\mathrm{N} / \mathrm{CS}=3.81^{\star}$ Isolation $+0.049^{\star} \mathrm{NSR}-0.040^{\star}$ Interaction -4.19 . Solid dots indicate values above the response surface; open circles indicate values below.

occur on open-ocean coral reefs, especially compared with enclosed harbors and disturbed embayments (Table 7). Paulay et al. (2002) determined that only $23 \%$ of the 85 introduced marine species found on Guam occurred outside Apra Harbor. Coles et al. (2003) found only six nonindigenous and cryptogenic species at coral reef sites on the 

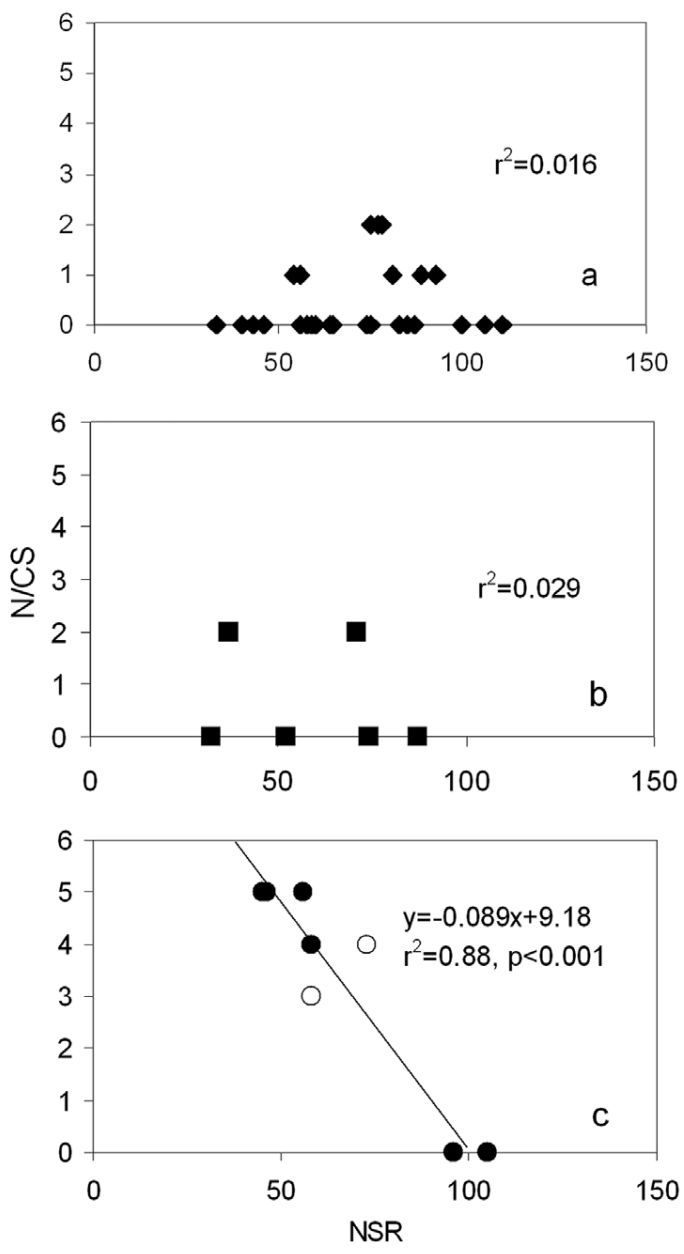

Figure 12. Comparisons of numbers of nonindigenous and cryptogenic species (N/CS) with native species richness (NSR) at different isolation index sites: $a$, open ocean; $b$, semiexposed coastlines; $c$, embayments (closed circles) and semienclosed harbors (open circles).

island of Tutuila, American Samoa, compared with 28 nonindigenous and cryptogenic species within Pago Pago Harbor. Similarly, in contrast to the approximately 100 nonindigenous and cryptogenic species previously recorded from $\mathrm{O}^{\prime}$ ahu harbors and in Kāne'ohe Bay (Coles et al. 1999a,b, 2002a) our rapid assessments detected a total of 26 nonindigenous and cryptogenic species (including three algae and four fishes) at 41 reef sites throughout the main Hawaiian Is- lands, with a maximum of six nonindigenous and cryptogenic species at any one site. Including the organisms identified from collections at seven reef sites nearest harbors, the total increased to 54 nonindigenous and cryptogenic species and a maximum of 23 at any one site. In contrast to the usual circumstances in Hawaiian harbors, where invertebrate nonindigenous and cryptogenic species are often among the most common or dominant species, this was not the case at any reef site. The alga Acanthophora spicifera (Vahl) Boerg was abundant on Moloka'i at Pūko'o (MO1) and at the Hotel Moloka'i (MO8) site; on Maui at Kahekili Beach Park (MA1), Papa'ula Point (MA3), and Mā'alaea Reef (MA7); and on O'ahu at the Nānākuli Point site (OA3). Another alga, Kappaphycus cf. alvarezii (Doty) Doty, was abundant at the two Kāne'ohe Bay sites (OA1 and OA2), and the ta'ape, Lutjanus kasmira, was abundant at 10 sites throughout the survey. The only invertebrate nonindigenous and cryptogenic species abundant at any site was the octocoral Carijoa riisei (Duchassaing \& Michelotti), which occurred on the undersides of concrete posts and metal sheet pilings of a collapsed dock adjacent to the coral reef at Māla Wharf, Maui (MA6). Remarkably, this species was absent from KA5 on Kaua'i, within the breakwater of Port Allen Harbor and only $600 \mathrm{~m}$ from where the octocoral was very abundant on the harbor's main dock pier pilings (Coles et al. 2004b). All other invertebrate nonindigenous and cryptogenic species were rare at all sites and usually consisted of a single or few individuals or colonies in cryptic locations.

Rapid assessments detected one to six introduced or cryptogenic species at the seven stations where collections and laboratory identifications yielded 5-21 species. By station, rapid assessments detected $8-43 \%$ of the numbers of micro- and macrobiota nonindigenous and cryptogenic species determined from laboratory identification, with a mean for the seven stations of $22.7 \%$. Conversely, one to four nonindigenous and cryptogenic species (two invertebrates and two fishes) detected by rapid assessment were not reported by laboratory identifications. Rapid 
TABLE 7

Numbers of Marine Nonindigenous (N), Cryptogenic (C), and Total Taxa Determined from the Hawaiian Islands, Johnston Atoll, Guam, and American Samoa

\begin{tabular}{|c|c|c|c|c|c|c|}
\hline Location & $\mathrm{N}$ & $\mathrm{C}$ & $\begin{array}{c}\text { Total } \\
\text { N/CS } a\end{array}$ & $\begin{array}{l}\text { Total } \\
\text { Taxa }\end{array}$ & $\begin{array}{c}\% \\
\text { N/CS }\end{array}$ & Source \\
\hline \multicolumn{7}{|l|}{ Harbors and embayments } \\
\hline O‘ahu, Pearl Harbor & 69 & 26 & 95 & 419 & 23.0 & Coles et al. $1997,1999 b$ \\
\hline O'ahu, commercial and small boat harbors & 73 & 27 & 100 & 585 & 17.0 & Coles et al. $1999 a$ \\
\hline Kāne'ohe Bay & 82 & 34 & 116 & 617 & 14.5 & Coles et al. $2002 a$ \\
\hline Hawaiian Neighbor Island harbors & 72 & 32 & 104 & 694 & 14.9 & Coles et al. $2004 b$ \\
\hline Guam, Apra Harbor & 27 & 29 & 46 & 682 & 6.7 & Paulay et al. 2002 \\
\hline Pago Pago Harbor, American Samoa & 17 & 11 & 28 & 977 & 2.9 & Coles et al. 2003 \\
\hline \multicolumn{7}{|l|}{ Coral reefs } \\
\hline Waikīkī & 19 & 33 & 52 & 617 & 6.9 & Coles et al. $2002 b$ \\
\hline Kaho'olawe & 3 & 0 & 3 & 298 & 1.0 & Coles et al. 1998 \\
\hline Midway Atoll & 4 & 0 & 4 & 444 & 1.5 & DeFelice et al. 1998 \\
\hline Johnston Atoll & 5 & 5 & 10 & 668 & 1.5 & Coles et al. 2001 \\
\hline French Frigate Shoals & 2 & 0 & 2 & 617 & 0.3 & DeFelice et al. 2002 \\
\hline Guam & 41 & 44 & 85 & 2878 & 1.5 & Paulay et al. 2002 \\
\hline Tutuila, American Samoa & 2 & 5 & 7 & 828 & 0.8 & Coles et al. 2003 \\
\hline Hawaiian Neighbor Islands, near harbors & 42 & 23 & 65 & 814 & 7.9 & Coles et al. $2004 b$ \\
\hline Hawaiian Neighbor Islands, 41 reef sites & 18 & 8 & 26 & 486 & 5.3 & This study \\
\hline
\end{tabular}

${ }^{a}$ N/CS, nonindigenous and cryptogenic species.

assessments were therefore clearly less sensitive for determining a full complement of introduced species that includes minute organisms and species in cryptic habitats. However, rapid assessments were more time and resource efficient, requiring about $1 \mathrm{hr}$ in the field and about $1 \mathrm{hr}$ for data entry per site. By comparison, although the time required for sample treatment, sorting, species identification, and verification by taxonomic experts varied substantially with organism abundance and species richness, about 1 month per station would be a conservative estimate, based upon this and previous studies on O'ahu.

Some sites near harbors or boat ramps were among those with the maximum numbers of nonindigenous and cryptogenic species, suggesting that harbors and boat ramps could act as sources or vectors for the spread of introduced species. This would be the expected pattern if boat transport of nonindigenous and cryptogenic species were the primary factor in determining their recruitment and final distribution. However, this pattern did not hold for the full array of rapid assessment sites, and some stations near har- bors or boat ramps had some of the lowest values for nonindigenous and cryptogenic species. This indicates that if these potential sources are important in the spread and proliferation of nonindigenous and cryptogenic species, their influence was not spatially consistent, nor did their influence extend very far. Many sites within a few hundred meters from harbors and docks showed few or no nonindigenous and cryptogenic species, and none exceeded a total of six species detected by rapid assessment or 23 by laboratory identification. No other factors considered likely to propagate or support nonindigenous and cryptogenic species (i.e., proximity to shorelines or streams mouths, presence of humanmade structures in the water, development or alteration of the shoreline, and apparent declines in reef condition that would suggest environmental disturbance) showed any significant relationship with numbers of nonindigenous and cryptogenic species detected by rapid assessment.

The most clearly defined relationship between nonindigenous and cryptogenic species determined from rapid assessment and any 
predictor variable was the degree to which the sites were isolated from the open ocean. This factor alone accounted for $45 \%$ of the variance in nonindigenous and cryptogenic species numbers. This finding agrees with observations in harbors and embayments on O'ahu (Coles et al. 1997, 1998, 1999a,b, $2002 a, b)$, which found decreasing numbers of nonindigenous and cryptogenic species in the more ocean-exposed areas of the study areas. Increasing ocean exposure also correlated with increasing native species richness. These relationships were also apparent on Tutuila, American Samoa (Coles et al. 2003), where nonindigenous and cryptogenic species ranged from 5 to 17 per site in the semienclosed inner Pago Pago Harbor to 1 to 4 nonindigenous and cryptogenic species per site on reefs distant from the harbor. By comparison, total taxa there were $102-185$ per site at the inner harbor sites and 403-449 per site on reefs outside the harbor.

Previous studies in Hawai'i (Coles et al. 1999a, 2002a, Coles and Eldredge 2002) have concluded that coral reef systems may be resistant to species introductions and disruptions of native populations. Paulay et al. (2002) concluded that the results of their study of introduced marine species in Guam supported the hypothesis articulated by Vermeij (1991) that diverse communities are more difficult to invade. Increased species richness of sessile organisms has been empirically determined to significantly decrease invasion success in coastal New England habitats (Stachowicz et al. 1999). Some studies in Australia also suggest that higher diversity of native biota in reef systems offer fewer opportunities for successful proliferation of new arrivals than is the case for lower-diversity temperate areas (Hutchings et al. 2002). However, Hewitt (2002) did not find a significant negative relationship between native species richness and introduced species for eight port surveys in tropical to temperate waters around Australia, although numbers of introduced species did increase significantly with latitude.

Similarly, in this survey we did not find an anticipated decrease of nonindigenous and cryptogenic species with increasing native species richness. Rather, a highly significant interaction term between ocean isolation and native species richness suggests that the association between the number of nonindigenous species and native species richness may be related to the degree of isolation from open-ocean exposure, with nonindigenous and cryptogenic species decreasing as native species richness increases in semiexposed environments and embayments.

In conclusion, this and previous surveys of nonindigenous species in Hawai'i indicate that, overall, few introduced marine invertebrates have colonized Hawai'i's coral reefs and even fewer are invasive (i.e., monopolizing habitats or displacing native species on reefs). Our results also suggest that the competition between native and nonindigenous species may be influenced by the degree to which the reef or habitat is isolated from open-ocean circulation, in interaction with the species richness of the native biota.

\section{ACKNOWLEDGMENTS}

We thank the Hawai'i Coral Reef Initiative director and staff for their guidance and assistance. Victor Bonito provided valuable assistance in the field for the Moloka'i survey. The Hawai'i State Department of Land and Natural Resources, Division of Aquatic Resources generously provided logistic support and boat transport on the islands of Maui, Hawai' $\mathrm{i}$, and $\mathrm{O}^{\prime} \mathrm{ahu}$, and we want to especially thank Paul Murakawa, Tony Montgomery, Skippy Hau, Russell Sparks, and Bill Walsh for providing this service. We are also grateful to the Hawai'i Institute of Marine Biology and the University of Hawai'i Zoology Department for providing logistic support and boat usage to access the Kanne'ohe Bay sites. Comments by three anonymous reviewers were very helpful in improving the paper's content and clarity. Specimens of taxa that were possible new introductions or that required species identification verification were sent to or seen by the following experts, who are gratefully acknowledged for their assistance-sponges: Ralph DeFelice, Los Angeles County Museum of Natural History; hydrozoans: Dale Calder, Royal Ontario Mu- 
seum, Toronto, Canada; vermetid gastropod mollusks: Anuschka Fauci, Department of Zoology, University of Hawai'i; bryozoans: Chela Zabin, Department of Zoology, University of Hawai' $i$; ascidians: Scott Godwin, Department of Natural Sciences, Bishop Museum, Honolulu, Hawai'i.

\section{Literature Cited}

Abbott, D. P., A. T. Newberry, and K. M. Morris. 1997. Section 6B: Ascidians. Bishop Mus. Spec. Publ. 64 (6B).

Bax, N., J. T. Carlton, A. Mathews-Amos, R. L. Haedrich, F. G. Howarth, J. E. Purcell, J. E. Rieser, and A. Gray. 2001. Conserving marine diversity through the control of biological invasions. Conserv. Biol. 451:145-176.

Birkeland, C. 2004. Ratcheting down coral reefs. BioScience 54:1021-1027.

Brock, V. E. 1960. The introduction of aquatic animals into Hawaiian waters. Int. Rev. Ges. Hydrobiol. 45:463-480.

Brown, E., E. Cox, P. Jokiel, K. Rodgers, W. Smith, B. Tissot, S. L. Coles, and J. Hultquist. 2004. Development of benthic sampling methods for the Coral Reef Assessment and Monitoring Program (CRAMP) in Hawai'i. Pac. Sci. 58:145158.

Carlton, J. T. 1985. Transoceanic and interoceanic dispersal of coastal marine organisms: The biology of ballast water. Oceanogr. Mar. Biol. Annu. Rev. 23:313371.

1994. Biological invasions and biodiversity in the sea: The ecological and human impacts of nonindigenous marine and estuarine organisms. Pages 5-11 in Nonindigenous estuarine and marine organisms (NEMO), Proceedings of the Conference and Workshop. Government Printing Office No. 0208-C-04, Seattle, Washington.

. 1996. Biological invasions and cryptogenic species. Ecology 77:1653-1655.

Carlton, J. T., and J. B. Geller. 1993. Ecological roulette: The global transport of nonindigenous marine organisms. Science (Washington, D.C.) 261:78-82.
Carlton, J. T., J. K. Thompson, L. E. Schemel, and F. H. Nichols. 1990. Remarkable invasion of San Francisco Bay (California, USA) by the Asian clam Potamocorbula amurensis. I. Introduction and dispersal. Mar. Ecol. Prog. Ser. 66:81-94.

Chapman, J. W., and J. T. Carlton. 1991. A test of criteria for introduced species: The global invasion by the isopod Synidotea laevidorsalis (Miers, 1881). J. Crustacean Biol. 11 (3): 386-400.

Cohen, A. N., J. T. Carlton, and M. C. Fountain. 1995. Introduction, dispersal and potential impacts of the green crab Carcinus maenas in San Francisco Bay, California. Mar. Biol. (Berl.) 122:225-237.

Coles, S. L., and H. Bolick. 2006. Assessment of invasiveness of the orange keyhole sponge Mycale armata in Kāne'ohe Bay, O'ahu, Hawai'i. Final Report Year 1. Contribution No. 2006-02 to the Hawai'i Biological Survey, Bishop Museum, Honolulu. http://hbs.bishopmuseum.org/pdf/ HCRI-report2006.pdf.

Coles, S. L., R. C. DeFelice, and L. G. Eldredge. 1999a. Nonindigenous marine species introductions in the harbors of the south and west shores of O'ahu, Hawai'i. Bishop Mus. Tech. Rep. 15. http://hbs .bishopmuseum.org/pdf/southshore.pdf.

_. 2002a. Nonindigenous species in Kān'eohe Bay, O'ahu, Hawai'í Bishop Mus. Tech. Rep. 24. http://hbs .bishopmuseum.org/pdf/kbay-report.pdf.

- 2002b. Nonindigenous marine species introductions at Waikīkī and Hawai' $i$ Kai, O'ahu, Hawai'i. Bishop Museum Tech. Rep. 25. http://hbs.bishopmuseum .org/pdf/waikiki.pdf.

Coles, S. L., R. C. DeFelice, L. G. Eldredge, and J. T. Carlton. 1997. Biodiversity of marine communities in Pearl Harbor, O'ahu, Hawai'i with observations on introduced species. Bishop Mus. Tech. Rep. 10. http://hbs.bishopmuseum.org/ pdf/PHReport.pdf.

. 1999b. Historical and recent introductions to non-indigenous marine species into Pearl Harbor, Oahu, Hawaiian Islands. Mar. Biol. (Berl.) 135:147-158.

Coles, S. L., R. C. DeFelice, and D. Minton. 
2001. Marine species survey of Johnston Atoll June 2000. U.S. Fish and Wildlife Service, Pacific Islands Area Office. Bishop Mus. Tech. Rep. 19. http://hbs.bishopmuseum .org/pdf/johnstonreport.pdf.

Coles, S. L., R. C. DeFelice, J. E. Smith, D. Muir, and L. G. Eldredge. 1998. Determination of baseline conditions for introduced marine species in nearshore waters of the island of Kaho'olawe, Hawai'i. Bishop Mus. Tech. Rep. 14. http://hbs .bishopmuseum.org/pdf/kahoolawe.pdf.

Coles, S. L., and L. G. Eldredge. 2002. Nonindigenous species introductions on coral reefs: A need for information. Pac. Sci. 56:191-209.

Coles, S. L., L. G. Eldredge, F. Kandel, P. A. Reath, and K. Longenecker. 2004a. Assessment of nonindigenous species on coral reefs in the Hawaiian Islands, with emphasis on introduced invertebrates. Bishop Mus. Tech. Rep. 27. http://hbs .bishopmuseum.org/pdf/hcri-2004.pdf.

Coles, S. L., P. A. Reath, K. Longenecker, H. Bollick, and L. G. Eldredge. 2004b. Assessment of nonindigenous marine species in harbors and on nearby coral reefs on Kaua'i, Moloka'i, Maui and Hawai'i. Bishop Mus. Tech. Rep. 29. http://hbs .bishopmuseum.org/pdf/tr29.pdf.

Coles, S. L., P. A. Reath, P. A. Skelton, V. Bonito, R. C. DeFelice, and L. Basch. 2003. Introduced marine species in Pago Pago Harbor, Fagatele Bay and the National Park Coast, American Samoa. Bishop Mus. Tech. Rep. 26. http://www.bishopmuseum .org/research/pbs/pdf/pagopago.pdf.

Daehler, C. C., and D. R. Strong. 1996. Status, prediction and prevention of introduced cordgrass Spartina spp. invasions in Pacific estuaries, USA. Biol. Conserv. 78:51-58.

DeFelice, R. C., S. L. Coles, D. Muir, and L. G. Eldredge. 1998. Investigation of the marine communities of Midway Harbor and adjacent lagoon, Midway Atoll, Northwestern Hawaiian Islands. Bishop Museum, Hawai'i Biological Survey Contr. No. 1998-014.

DeFelice, R., D. Minton, and S. Godwin. 2002. Records of shallow-water marine in- vertebrates from French Frigate Shoals, Northwestern Hawaiian Islands with a note on nonindigenous species. Bishop Mus. Tech. Rep. 23.

Eldredge, L. G., and J. T. Carlton. 2002. Hawaiian marine bioinvasions: A preliminary assessment. Pac. Sci. 56:211-212.

Englund, R. A., K. Arakaki, D. J. Preston, S. L. Coles, and L. G. Eldredge. 2000. Nonindigenous freshwater and estuarine species introductions and their potential to affect sportfishing in the lower stream and estuarine regions of the south and west shores of O'ahu, Hawai'i. Bishop Mus. Tech. Rep. 17.

Gosliner, T. M. 1995. Introduction and spread of Philine auriformis (Gastropoda: Opisthobranchia) from New Zealand to San Francisco Bay and Bodega Harbor. Mar. Biol. (Berl.) 122:249-255.

Greenberg, N., R. L. Garthwaite, and D. C. Potts. 1996. Allozyme and morphological evidence for a newly introduced species of Aurelia in San Francisco Bay, California. Mar. Biol. (Berl.) 125:401-410.

Grigg, R. W. 2003. Invasion of a deep black coral bed by an alien species, Carijoa riisei, off Maui, Hawaii. Coral Reefs 22:121122.

- 2004. Harvesting impacts and invasion by an alien species decrease estimates of black coral yield off Maui, Hawai'i. Pac. Sci. 58:1-6.

Hewitt, C. L. 2002. Distribution and diversity of Australian tropical marine bioinvasions. Pac. Sci. 56:213-222.

Hewitt, C. L., M. L. Campbell, K. M. Moore, N. B. Murfet, and B. Robertson. 1998. Introduced species survey. Port of Hay Point, Queensland. CSIRO Centre for Research on Introduced Marine Pests, Report for the Ports Corporation of Queensland, Brisbane.

Hoedt, F. E., J. H. Choat, J. Collins, and J. J. Cruz. 2000. Mourilyan Harbour and Abbot Point surveys: Port marine baseline surveys and surveys for introduced marine pests. School of Marine Sciences and Aquaculture, James Cook University, Report for the Ports Corporation of Queensland, Brisbane. 
2001a. Port of Lucinda surveys: Port marine baseline surveys and surveys for introduced marine pests. School of Marine Sciences and Aquaculture, James Cook University, Report for the Ports Corporation of Queensland, Brisbane.

Hoedt, F. E., J. H. Choat, J. J. Cruz, K. M. Neil, and J. Collins. 2001b. Survey of the Port of Weipa: Baseline surveys and surveys for introduced marine pests. School of Marine Sciences and Aquaculture, James Cook University, Report for the Ports Corporation of Queensland, Brisbane.

Hutchings, P. A., R. W. Hilliard, and S. L. Coles. 2002. Species introductions and potential for marine pest invasions into tropical marine communities, with special reference to the Indo-Pacific. Pac. Sci. $56: 223-233$.

Jokiel, P. L. 2002. Hawaici Coral Reef Assessment and Monitoring Program (CRAMP). Hawai'i Coral Reef Initiative final report. http://www.hawaii.edu/ssri/ hcri/files/jokiel_final_report_00-01.pdf.

Kinzie, R. A., III. 1968. The ecology of the replacement of Pseudosquilla ciliata by Gonodactylus falcatus (Crustacea: Stomatopoda) recently introduced into the Hawaiian Islands. Pac. Sci. 22:465-474.

- 1984. Aloha also means goodbye: A cryptogenic stomatopod in Hawaii. Pac. Sci. 38:298-311.

Maciolek, J. A. 1984. Exotic fishes in Hawaii and other islands in Oceania. Pages 131161 in R. Courtenay Jr. and J. R. Stauffer Jr., eds. Distribution, biology and management of exotic fishes. Johns Hopkins Press, Baltimore.

Mills, C. E., and F. Sommer. 1995. Invertebrate introductions in marine habitats: Two species of hydromedusae (Cnidaria) native to the Black Sea, Maeotias inexspectata and Blackfordia virginica, invade San Francisco Bay. Mar. Biol. (Berl.) 122:279288.

Neil, K. M., F. E. Hoedt, J. Sheaves, J. J. Cruz, and J. H. Choat. 2001. Survey of the Port of Karumba: Port marine surveys and surveys for introduced marine pests. James Cook University, CRC Reef Re- search Centre Report for the Ports Corporation of Queensland, Brisbane.

Paulay, G., L. Kirkendale, G. Lambert, and C. Meyer. 2002. Anthropogenic biotic interchange in a coral reef ecosystem: A case study from Guam. Pac. Sci. 56:403-422.

Randall, J. E. 1987. Introductions of marine fishes to the Hawaiian Islands. Bull. Mar. Sci. 41:490-502.

Randall, J. E., and R. K. Kanayama. 1972. Hawaiian fish immigrants. Sea Front. 18:144-153.

Rodgers, S. K., and E. F. Cox. 1999. Rate of spread of introduced rhodophytes Kappaphycus alvarezii, Kappaphycus striatum, and Gracilaria salicornia and their current distributions in Kāne'ohe Bay, O'ahu, Hawai'i. Pac. Sci. 53:232-241.

Ruiz, G. M., J. T. Carlton, E. D. Grosholz, and A. H. Hines. 1997. Global invasions of marine and estuarine habitats by nonindigenous species: Mechanisms, extent and consequences. Am. Zool. 31:621-632.

Ruiz, G. M., P. W. Fofonoff, M. J. Wonham, and A. H. Hines. 2000. Invasion of coastal marine communities in North America: Apparent patterns, processes, and biases. Annu. Rev. Ecol. Syst. 31:481-531.

Russell, B. C., and C. L. Hewitt. 2000. Baseline survey of the port of Darwin for introduced marine species. Northern Territory Department of Transport and Works Marine Branch, Darwin, Northern Territory, Australia.

Smith, J. E., C. L. Hunter, and C. M. Smith. 2002. Distribution and reproductive characteristics of nonindigenous and invasive marine algae in the Hawaiian Islands. Pac. Sci. 56:299-315.

Stachowicz, J. J., R. B. Whitlatch, and R. W. Osman. 1999. Species diversity and invasion resistance in a marine ecosystem. Science (Washington, D.C.) 286:15771579.

Tissot, B. N., W. A. Walsh, and L. E. Hallacher. 2001. The effectiveness of marine protected areas and the impacts of aquarium fish collecting in Hawai'i. Hawai'i Coral Reef Initiative final report year 2001. http://www.hawaii.edu/ssri/ hcri/files/tissot_final_report_00-01.pdf. 
Vermeij, G. J. 1991. When biotas meet: Understanding biotic interchange. Science (Washington, D.C.) 253:1099-1104.

Woo, M., C. M. Smith, and W. Smith. 1999. Ecological interactions and impacts of in- vasive Kappaphycus striatum in Kāne'ohe Bay, a tropical reef. Pages 186-191 in J. Pederson, ed. Marine bioinvasions. MIT Sea Grant Program, Cambridge, Massachusetts. 
